\title{
Microstructures, mechanical, and biological properties of a novel Ti-6V-4V/zinc surface nanocomposite prepared by friction stir processing
}

This article was published in the following Dove Press journal: International Journal of Nanomedicine

Chenyuan Zhu, ${ }^{1,2, *}$ Yuting Lv, ${ }^{3,4, *}$ Chao Qian, ${ }^{1,2, *}$ Zihao Ding, ${ }^{3,5}$ Ting Jiao, ${ }^{1,2}$ Xiaoyu $\mathrm{Gu},{ }^{1,2}$ Eryi Lu, ${ }^{6}$ Liqiang Wang, ${ }^{3}$ Fuqiang Zhang ${ }^{1,2}$

'Department of Prosthodontics, Ninth People's Hospital, Shanghai Jiao Tong University School of Medicine, ${ }^{2}$ Shanghai Key Laboratory of Stomatology, Shanghai Research Institute of Stomatology, National Clinical Research Center of Stomatology, ${ }^{3}$ State Key Laboratory of Metal Matrix Composites, Shanghai Jiao Tong University, Shanghai, ${ }^{4}$ College of Mechanical and Electronic Engineering, Shandong University of Science and Technology, Qingdao, People's Republic of China; ${ }^{5}$ Department of Materials Science and Engineering, Carnegie Mellon University, Pittsburgh, PA, USA; ${ }^{6}$ Department of Stomatology, Ren Ji Hospital, Shanghai Jiao Tong University School of Medicine, Shanghai,

People's Republic of China

*These authors contributed equally to this work

Correspondence: Liqiang Wang State Key Laboratory of Metal Matrix Composites, Shanghai Jiao Tong University, 800 Dongchuan Road, Shanghai 200240, People's Republic of China Email wang_liqiang@sjtu.edu.cn

\section{Fuqiang Zhang}

Shanghai Key Laboratory of Stomatology, Shanghai Research Institute of Stomatology, National Clinical Research Center of Stomatology, No 639.

Zhizaoju Road, Shanghai 2000II, People's Republic of China

Email fqzhang1226@163.com
Background: The interaction between the material and the organism affects the survival rate of the orthopedic or dental implant in vivo. Friction stir processing (FSP) is considered a new solid-state processing technology for surface modification.

Purpose: This study aims to strengthen the surface mechanical properties and promote the osteogenic capacity of the biomaterial by constructing a Ti-6Al-4V (TC4)/zinc (Zn) surface nanocomposites through FSP.

Methods: FSP was used to modify the surface of TC4. The microstructures and mechanical properties were analyzed by scanning electron microscopy, transmission electron microscopy, nanoindentation and Vickers hardness. The biological properties of the modified surface were evaluated by the in vitro and in vivo study.

Results: The results showed that nanocrystalline and numerous $\beta$ regions, grain boundary $\alpha$ phase, coarser acicular $\alpha$ phase and finer acicular martensite $\alpha^{\prime}$ appeared because of the severe plastic deformation caused by FSP, resulting in a decreased elastic modulus and an increased surface hardness. With the addition of Zn particles and the enhancement of hydrophilicity, the biocompatibility was greatly improved in terms of cell adhesion and proliferation. The in vitro osteogenic differentiation of rat bone marrow stromal cells and rapid in vivo osseointegration were enhanced on the novel TC4/Zn metal matrix nanocomposite surface.

Conclusion: These findings suggest that this novel TC4/Zn surface nanocomposite achieved by FSP has significantly improved mechanical properties and biocompatibility, in addition to promoting osseointegration and thus has potential for dental and orthopedic applications.

Keywords: nanocomposite, friction stir processing, nanocrystalline/ultrafine grained, bone marrow stromal cells, cell proliferation, osteogenic differentiation

\section{Introduction}

For biomaterials used in human tissue and organ regeneration, the interaction between the material and the organism affects the survival rate of the orthopedic or dental implant in vivo. For example, the implant may cause side effects in the organism, including cytotoxicity, inflammation, and anaphylaxis. Additionally, corrosion and abrasion will gradually occur during the service lifetime of the implant, leading to a decline in its mechanical properties. ${ }^{1,2}$ In complex biomechanical environments, ideal metal implants should not only possess excellent mechanical properties but also be biocompatible. In recent years, titanium (Ti) alloys have been widely used in various fields, including orthopedics, dental implantation, and tissue engineering, because of their good fatigue resistance, high corrosion resistance, and good biocompatibility. Many commercial products for replacing body parts or functions, such as artificial 
joint replacements, bone trauma products, and oral implants, have been fabricated from Ti alloys..$^{3-5}$

Among all Ti alloys, Ti-6Al-4V (TC4) is the most typical and the most frequently used $\alpha+\beta$ type Ti due to its high strength-to-weight ratio, low density, high corrosion resistance, and biocompatibility. ${ }^{6,7}$ Nevertheless, when the metal is implanted in a living body, it inevitably releases ions into the saline solution of bodily fluids. ${ }^{8}$ When TC4 is used as an implant material, aluminum (Al) and vanadium (V) ions released into surrounding tissues can adversely affect the body. ${ }^{2,9,10}$ Moreover, the difference between the elastic modulus of the biomaterial and that of the surrounding hard tissue can result in a stressshielding effect. ${ }^{11,12}$ To avoid this disadvantage of TC4 without changing the composition of the whole material, we designed a surface modification process to create a nanocomposite layer on the implant surface, thereby improving its biocompatibility while largely maintaining its mechanical properties.

Friction stir processing (FSP) is considered as a new solidstate processing technology for surface modification. ${ }^{13,14}$ During FSP, a probe presses into a metallic substrate and rotates and moves along the processing direction. The friction between the probe and base material $(\mathrm{BM})$ provides a heat source to increase the temperature of the metallic substrate. The heat input is accompanied by severe plastic deformation, resulting in recrystallization and the evolution of texture within the stirred zone (SZ) and coarsening within and around the SZ. Based on microstructural characterization, four zones can be distinguished in FSPed metal, the SZ, the thermo-mechanically affected zone (TMAZ), the heat-affected zone (HAZ), and the $\mathrm{BM}$ zone. These various zones show different microstructures, leading to different mechanical properties. ${ }^{15}$ A new metal matrix nanocomposite (MMNC) structure is achieved by adding reinforcement particles or metal powder into the metallic substrate during plastic flow for participation in grain refinement, metallurgical bond behavior, and plastic deformation. The addition of different elements can promote the evolution of different microstructures, thereby optimizing the surface properties.

Recently, FSP has been increasingly applied to medical Ti. $\mathrm{Li}$ et $\mathrm{al}^{7}$ reported that the hardness and wear performance of the TC4 alloy could be significantly improved by FSP. Wang et $\mathrm{al}^{16}$ investigated superelasticity in a novel TiNbTaZr alloy prepared by FSP, providing a new way to obtain desired properties for biomedical applications. In our previous work, ${ }^{17}$ we prepared a $\mathrm{Ti} / \mathrm{SiC} \mathrm{MMNC}$ by adding $\mathrm{SiC}$ particles into the Ti during FSP and reported that the grain-refined surface promoted cell adhesion and osteogenic differentiation of rat bone marrow stromal cells (BMSCs). ${ }^{18}$ Compared with the coarse-grained surface, the ultrafine-grained Ti surface exhibited improved corrosion resistance..$^{19,20}$ Estrin et $\mathrm{al}^{21,22}$ have also demonstrated that ultrafine-grained surface can promote cell adhesion and proliferation of stem cells and osteoblasts.

The surface properties of a biomaterial directly affect early cell adhesion, which is the primary stage of the interaction between tissue and medical implants, followed by cell proliferation and differentiation. The osteogenic capacity and rapid osseointegration of a biomaterial play vital roles in determining the success rate of an implant. ${ }^{23,24}$ FSP was used in this study to modify the alloy surface and to add zinc ( $\mathrm{Zn}$ ) particles to optimize its surface properties and to improve its biocompatibility. In this study, Zn was chosen as the reinforcement particle because it is an essential trace element in the human body. Zn participates in the synthesis of various enzymes and plays an indispensable role in bone growth and the maintenance of physiological cellular functions. In addition, $\mathrm{Zn}$ oxide has excellent antimicrobial properties and elemental $\mathrm{Zn}$ can effectively promote the osteogenic differentiation of BMSCs. ${ }^{25-27}$

Therefore, we hypothesized that adding Zn to TC4 during FSP could decrease the grain size, lower the elastic modulus, improve the surface hardness, and optimize the biological properties to further satisfy the requirements of in vivo implants. Thus, this study aimed to fabricate a novel TC4/Zn MMNC surface using FSP to strengthen the surface properties and promote the osteogenic capacity of the material. The microstructures and mechanical properties and their relationship were thoroughly explored. In addition, the biological activity of the nanocomposite surface was investigated both in vitro and in vivo.

\section{Methods}

\section{Fabrication of TC4/Zn MMNC}

Commercially available $\mathrm{Zn}$ powder $(99.9 \%$ purity with an average particle size of $50 \mathrm{~nm}$; Shanghai Shuitian Materials Technology Co., Ltd., Shanghai, People's Republic of China) and TC4 plates (Gold Borui Titanium Industry Co., Ltd., Baoji, People's Republic of China) were used as raw materials in this study. The TC4 BM was used as the control group (Con). The TC4 MMNC that underwent FSP without the addition of $\mathrm{Zn}$ is denoted as FSP. The TC4/Zn MMNCs that underwent FSP with premade holes of different depths are denoted as Zn-I-FSP (1 mm holes) and Zn-II-FSP (0.5 mm holes). FSP was performed using a professional-grade friction-stir welding machine (CFSW-PL800; Beijing FSW Technology Co., Ltd., Beijing, People's Republic of China) with a tungsten steel FSP tool in an argon atmosphere to prevent oxidation. The probe was $10 \mathrm{~mm}$ in diameter, had a concave shoulder and a $2 \mathrm{~mm}$ pin height, and was tilted by $2.5^{\circ}$. When the parameters of the probe were defined, the volumetric ratio of the added $\mathrm{Zn}$ nanoparticles could be roughly estimated. The volumetric ratio of $\mathrm{Zn}$ in the $\mathrm{Zn}-\mathrm{I}-\mathrm{FSP}$ group was $\sim 5.1 \%$, 
whereas the ratio for the Zn-II-FSP group was $\sim 2.48 \%$. A constant traverse speed $(v)$ of $50 \mathrm{~mm} / \mathrm{min}$ at a rotation rate ( $\omega)$ of $300 \mathrm{rpm}$ was adopted to fabricate TC4/Zn MMNC by FSP. Single-pass FSP was conducted in this study. Samples with dimensions of $1 \times 1 \mathrm{~cm}$ were cut from the $S Z$ at the surface of the FSP plate for microstructural examination, mechanical testing, and cell experiments. Before making microstructural observations, samples were cut, ground, and polished according to standard procedures and were then etched with a solution consisting of $2 \% \mathrm{HF}$ and $6 \% \mathrm{HNO}_{3}$ for $5 \mathrm{~s} .{ }^{28-30}$ Longitudinal sections of the TC4/Zn MMNC were prepared using a wire cutting machine and were then thinned using an ion thinning instrument from the upper surface to the bottom. Cylindrical implants $2 \mathrm{~mm}$ in diameter and $6 \mathrm{~mm}$ in length were cut from the SZ using the wire cutting machine for the in vivo study.

\section{Microstructural change in TC4/Zn MMNC}

The microstructure of FSP-processed samples was analyzed by scanning electron microscopy (SEM) with a Quanta 200 microscope (FEI Company, Eindhoven, the Netherlands) at $20 \mathrm{kV}$. Transmission electron microscopy (TEM; JEM-200 EX; JEOL, Tokyo, Japan) was used to observe the microstructures of all FSP zones ranging from the upper surface to the bottom of the MMNC (0-1.5 mm). The relative surface wettability was estimated from the contact angle using a contact angle meter (Kruss $\mathrm{GmbH}$, Hamburg, Germany) with water as the medium.

\section{Surface properties of TC4/Zn MMNC}

Nanoindentation tests were performed using a NANO Indenter G200 Testing System with a diamond Berkovich tip with continuous loading up to a maximum of $10 \mathrm{mN}$. Before each unloading process, a $30 \mathrm{~s}$ dwell time was applied under a fixed load. Load and displacement were continuously monitored and recorded to plot a curve. Using the obtained data, the nanoindentation depth and elastic modulus of the material were calculated. The Vickers hardness was measured using a microhardness tester (Shanghai Taiming Optical Instrument Co., Ltd., Shanghai, People's Republic of China) under a load of $50 \mathrm{~g}$. Eleven samples were tested.

\section{BMSC adhesion and cell proliferation on TC4/Zn MMNC surface}

All animal procedures and experiments were approved by the Animal Research Committee of the Ninth People's Hospital affiliated with the Shanghai Jiao Tong University School of Medicine, of which the guidelines were followed for the welfare of the animals. BMSCs were isolated from the femurs and tibias of 6-week-old male Sprague Dawley (SD) rats, as previously reported. ${ }^{31}$ The cells were cultured in Dulbecco's
Modified Eagle's Medium (DMEM) with 10\% fetal bovine serum in an incubator with a $5 \% \mathrm{CO}_{2}$ atmosphere at $37^{\circ} \mathrm{C}$. Cell identification was conducted according to our previously published procedures. ${ }^{18}$ Cells at passage 2 or 3 were used in subsequent experiments.

Nuclei were stained during the initial seeding period (1, 4, and $24 \mathrm{~h}$ ) to count the adherent cells on the different samples. At each time point, nonadherent cells were removed using a phosphate-buffered saline (PBS) rinse. The cells were fixed with $4 \%$ paraformaldehyde for $30 \mathrm{~min}$ at $4^{\circ} \mathrm{C}$. Next, nuclei were stained with 4',6-diamidino-2-phenylindole (DAPI) for $10 \mathrm{~min}$ and the samples were then observed using a fluorescence microscope (IX71; Olympus Corporation, Tokyo, Japan). Cell counts were quantitatively analyzed using a Z2 Coulter particle count and size analyzer (Beckman Coulter, Brea, CA, USA).

Integrin $\beta 1$ immunofluorescence was detected to confirm the expression of this adhesion-related protein. The initial cell seeding density was $2.0 \times 10^{4}$ cells/well for $24 \mathrm{~h}$. After fixation with $4 \%$ paraformaldehyde for $30 \mathrm{~min}$ at $4{ }^{\circ} \mathrm{C}$, the samples were sequentially treated with $0.5 \%$ Triton X-100 and $3 \%$ bovine serum albumin (BSA) at room temperature (RT). A specific primary rabbit-anti-rat antibody targeting integrin $\beta 1$ (eBioscience Inc., San Diego, CA, USA) was then added and incubated with the cells for $8 \mathrm{~h}$ at $4^{\circ} \mathrm{C}$. The cells were subsequently incubated with a fluorescein isothiocyanate (FITC)-conjugated antirabbit IgG antibody (Jackson ImmunoResearch Laboratories Inc., West Grove, PA, USA) for $1 \mathrm{~h}$ at RT away from light. The cell cytoskeleton was stained with a tetramethylrhodamine (TRITC)-phalloidinconjugated antibody (KenGEN Biotech, Nanjing, People's Republic of China). Nuclei were stained with DAPI for $10 \mathrm{~min}$, and the samples were observed using a fluorescence microscope (IX71; Olympus, Tokyo, Japan).

An 3-(4,5)-dimethylthiahiazo (-z-y1)-3,5-diphenytetrazoliumromide (MTT) cell metabolic activity assay was used to measure mitochondrial activity to evaluate the proliferation and viability of BMSCs on the samples. First, cells at a density of $2.0 \times 10^{4}$ cells $/ \mathrm{mL}$ were plated onto each specimen in a 24-well plate. After culturing for 1, 4, 7, and 10 days, MTT solution was added to target wells. During $4 \mathrm{~h}$ of incubation, formazan was formed and then dissolved in dimethyl sulfoxide. Optical density (OD) absorbance values were measured at $490 \mathrm{~nm}$ using an ELX Ultra microplate reader (Bio-Tek, Winooski, VT, USA). The experiment was conducted in triplicate.

\section{BMSC osteogenic differentiation on the TC4/Zn MMNC}

Osteogenic differentiation was evaluated by alkaline phosphatase (ALP) staining, ALP activity, and real-time PCR. 
Cells at a density of $2.0 \times 10^{4}$ cells $/ \mathrm{mL}$ were plated onto each specimen in a 24 -well plate. On the 7 th and 14 th days of culturing, the cells on the target samples were fixed and stained with BCIP/NBT kit reagents (Beyotime, Shanghai, People's Republic of China) according to the manufacturer's instructions. For the quantitative ALP assay, the cells were lysed and incubated with $p$-nitrophenyl phosphate (Sigma-Aldrich Co., St Louis, MO, USA) at $37^{\circ} \mathrm{C}$ for $30 \mathrm{~min}$. ALP activity was estimated by the OD absorbance values at $405 \mathrm{~nm}$ and normalized to the total cellular protein. The total protein content was determined using a Bio-Rad protein assay kit (Bio-Rad Laboratories Inc., Hercules, CA, USA) using the OD absorbance values measured at $630 \mathrm{~nm}$. The experiment was conducted in triplicate.

The osteogenesis-related genes examined included Runx2, ALP, OSX,COL-1 $\alpha, O P N$, and $O C N$. Cells were seeded at a density of $2.0 \times 10^{4}$ cells $/ \mathrm{mL}$ on the Ti plates $(1 \times 1 \mathrm{~cm})$ and were cultured for 7 and 14 days. Each group included five specimens at each time point to ensure that there was sufficient total RNA for PCR. Total RNA was extracted using a TaKaRa MiniBEST Universal RNA Extraction kit (TaKaRa Bio Inc., Otsu, Japan) according to the manufacturer's instructions, and cDNA was synthesized with a TaKaRa PrimeScript ${ }^{\mathrm{TM}}$ 1st Strand cDNA Synthesis kit. The expression levels of the target genes were analyzed using a Bio-Rad Quantitative Real-Time PCR system (Bio-Rad Laboratories Inc.). The relative expression level was normalized to that of the internal control gene $\beta$-actin. The $\Delta \Delta \mathrm{CT}$ method was applied to calculate the target gene expression levels, and the data from the Con were used as the control. The experiment was conducted in triplicate.

\section{In vivo osseointegration of $\mathrm{TC} 4 / \mathrm{Zn}$ MMNC implant}

The surgical procedures were performed under sterile conditions. After anesthesia via injection with ketamine $(10 \mathrm{mg} / \mathrm{kg})$, the distal femur of 10 -week-old SD rats was carefully exposed through a skin incision and periosteum blunt dissection. A hole $(\varnothing=2.0 \mathrm{~mm})$ was drilled into the distal femur along the direction of the medullary cavity. Four types of implants $(\varnothing=2 \times 6 \mathrm{~mm})$ were randomly inserted into each of the holes, and the wounds were conventionally sutured. Each type of implant was tested in triplicate.

Microcomputed tomography (micro-CT), sequential fluorescent labeling observation, and Van Gieson's staining were used to evaluate new bone formation around the TC4/Zn MMNC implant in vivo. Sequential fluorescent labeling was used to evaluate the rate of new bone formation and mineralization around the TC4/Zn MMNC implant in vivo.
Then, at 2, 4, and 6 weeks after the operation, $25 \mathrm{mg} / \mathrm{kg}$ of tetracycline (TE; Sigma-Aldrich Co.), $30 \mathrm{mg} / \mathrm{kg}$ of alizarin red S (AL; Sigma-Aldrich Co.), and $20 \mathrm{mg} / \mathrm{kg}$ of calcein (CC; Sigma-Aldrich Co.) were injected intraperitoneally. At 8 weeks after operation, all SD rats were sacrificed and the femurs with the implants were harvested and fixed in $10 \%$ buffered formaldehyde. The micro-CT scanning parameters were set at $65 \mathrm{kV}$ using an $\mathrm{Al} 1 \mathrm{~mm}$ filter with a resolution of $18 \mathrm{~mm}$. The new bone organization was assessed by parameters including the trabecular number (Tb.N), bone volume (BV), and BV/total volume (TV), as analyzed by micro-CT. After micro-CT examination, all samples were dehydrated using graded ethanol, embedded in polymethylmethacrylate (PMMA), and cut into $150 \mu \mathrm{m}$ thick sections using a Leica SP1600 saw microtome (Leica Microsystems, Wetzlar, Germany). These sections were manually pasted on a slide and carefully polished to a final thickness of $\sim 40 \mu \mathrm{m}$. Sequential fluorescent labels were observed using confocal laser scanning microscopy (CLSM; Olympus Corporation). The percentage of the labeling area was calculated using ImageJ $1.48 \mathrm{v}$ (National Institutes of Health, Bethesda, MA, USA). The bone-implant contact (BIC) was calculated from the histomorphometric measurements by Van Gieson staining.

\section{Results}

\section{Microstructures of TC4/Zn MMNC}

Figure 1 shows SEM micrographs of the upper surface of the TC4/Zn MMNC prepared by FSP. As shown in Figure 1A and $\mathrm{B}$, the microstructures of the TC4 alloy are composed of prior $\alpha$ and $\beta$ phases and the $\beta$ particles are mainly located on grain boundaries (GBs) or at triple junctions of $\alpha$ grains. This is the typical microstructure of the TC4 alloy, which has been reported in many investigations. ${ }^{7,32,33}$ After FSP, numerous $\beta$ regions, GB $\alpha$ phase, coarser acicular $\alpha$ phase, and finer acicular martensite $\alpha^{\prime}$ appear (Figure 1C and D). The magnified image clearly shows that the grains are significantly refined after FSP (Figure 1D), with the simultaneous formation of finer lamellar $\alpha$ and $\beta$ phases. These results indicate that during FSP, the peak temperature of the TC4 alloy can reach the $\beta$ phase transus temperature due to severe plastic deformation and the alloy completely transforms to the $\beta$ phase. During the cooling process, the $\alpha$ phase precipitates first from the GB of the $\beta$ phase and then precipitates and grows within the $\beta$ phase. Meanwhile, martensite $\alpha^{\prime}$ is also formed due to the high cooling rate (Figure 1D). Martensite $\alpha^{\prime}$ has been reported to precipitate when the cooling rate is $>410^{\circ} \mathrm{C} / \mathrm{s}$ during FSP. ${ }^{7}$ The FSP and TC4/Zn MMNC groups exhibit similar microstructural features. However, the $\beta$ region is significantly larger after adding $\mathrm{Zn}$ to the TC4 

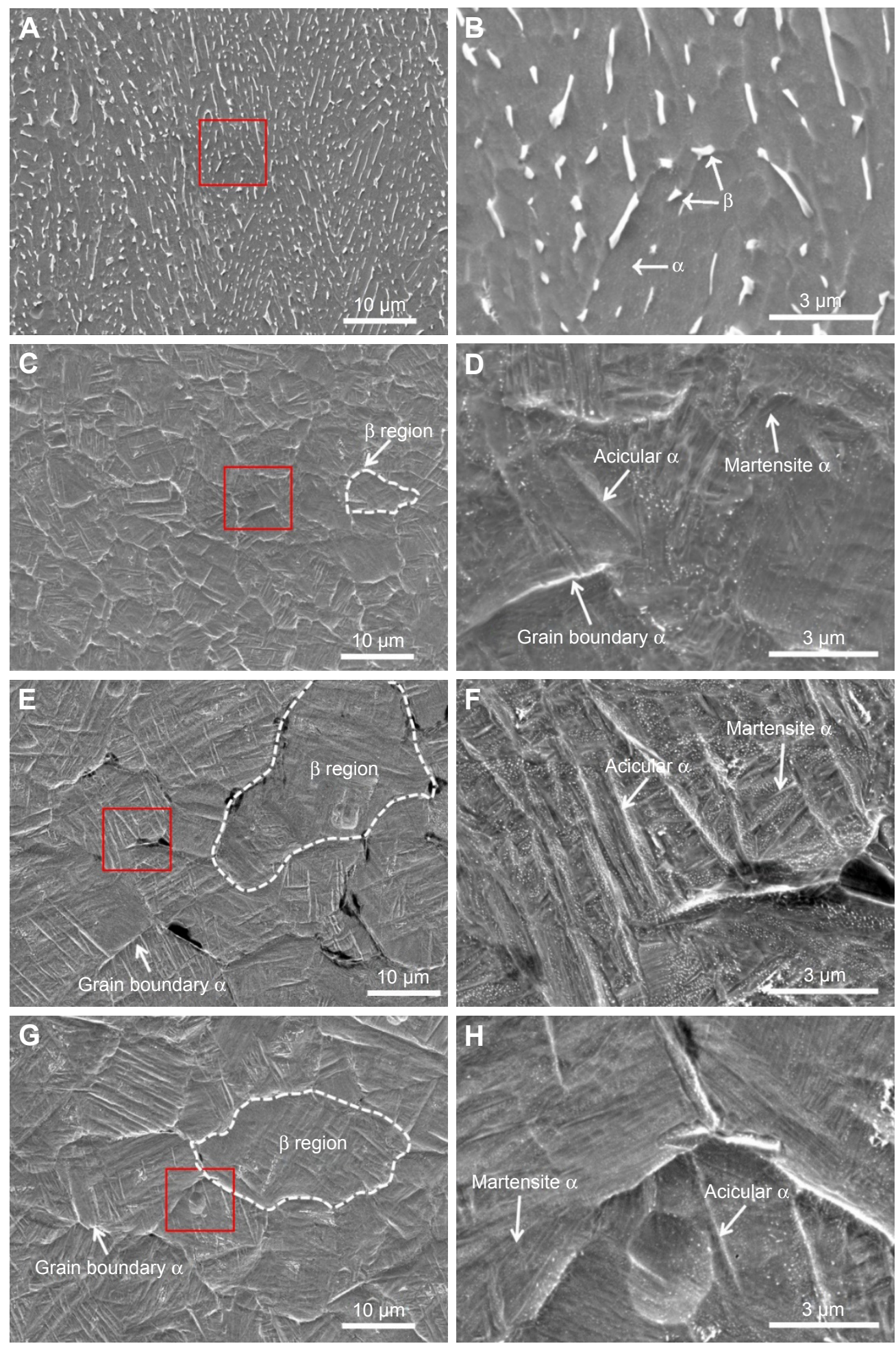

Figure I SEM micrographs showing the microstructures of the various alloys investigated in this study.

Notes: (A) Matrix TC4 alloy, (C) FSP group, (E) Zn-I-FSP group, and (G) Zn-II-FSP group. (B, D, F, and H) Magnified images of the red square regions in (A), (C), (E), and (G), respectively.

Abbreviations: FSP, friction stir processing; SEM, scanning electron microscopy; TC4, Ti-6Al-4V; Zn, zinc.

alloy (Figure 1C, E, and G). The size of the $\beta$ regions in the FSP, Zn-I-FSP and Zn-II-FSP groups is $\sim 10,20$, and $15 \mu \mathrm{m}$, respectively. Because the volumetric ratio of $\mathrm{Zn}$ in the $\mathrm{SZ}$ was controlled artificially via controlling the depth of the blind hole, the content of $\mathrm{Zn}$ in the $\mathrm{Zn}$-I-FSP group is higher than that in the $Z n-I I-F S P$ group. Thus, the $\beta$ phase region in the TC4/Zn nanocomposite grows with increasing $\mathrm{Zn}$ content. In addition, $\mathrm{Zn}$-rich particles are found in the nanocomposite, 
some of which are identified as $\mathrm{TiZn}_{2}$, indicating that $\mathrm{Zn}$ reacted with Ti and formed a new compound during FSP. Figure $1 \mathrm{~F}$ and $\mathrm{H}$ also clearly show that the quantity of $\mathrm{Zn}$-rich particles increases with the increasing $\mathrm{Zn}$ content.

To understand the microstructural evolution in detail, transverse cross-sections of the Zn-I-FSP group alloy were observed using TEM. The results revealed significant differences in the upper surface (within $200 \mu \mathrm{m}$ ) and the middle region of the SZ ( 1 $\mathrm{mm}$ from upper surface). Figure 2 shows TEM images of the upper surface in the Zn-I-FSP

A

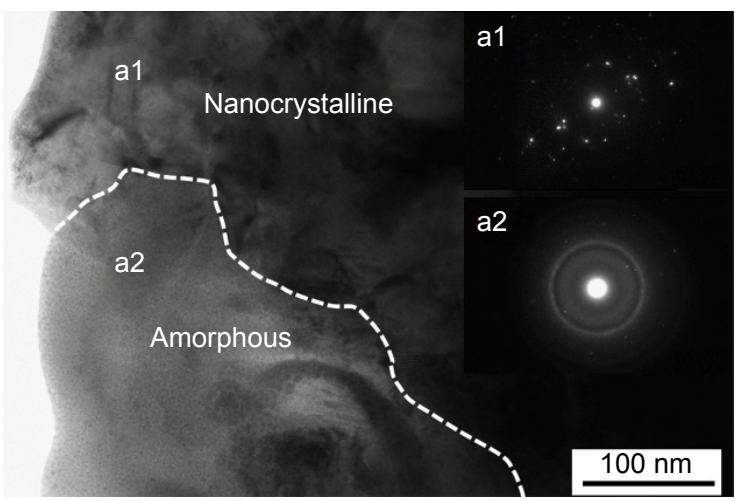

C

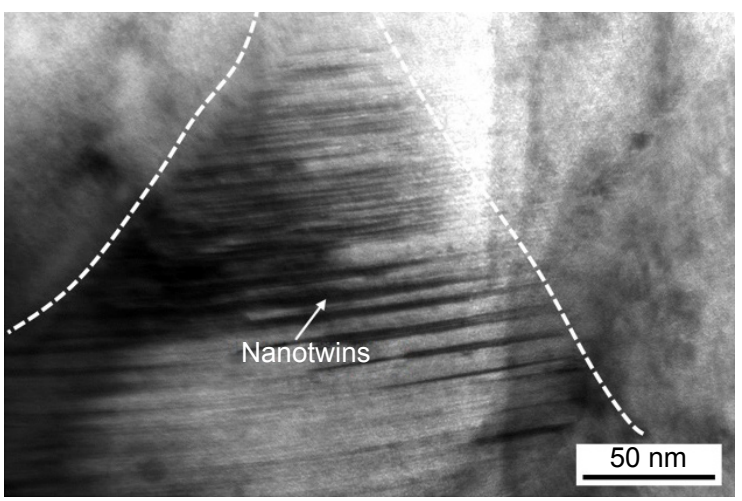

$\mathbf{E}$

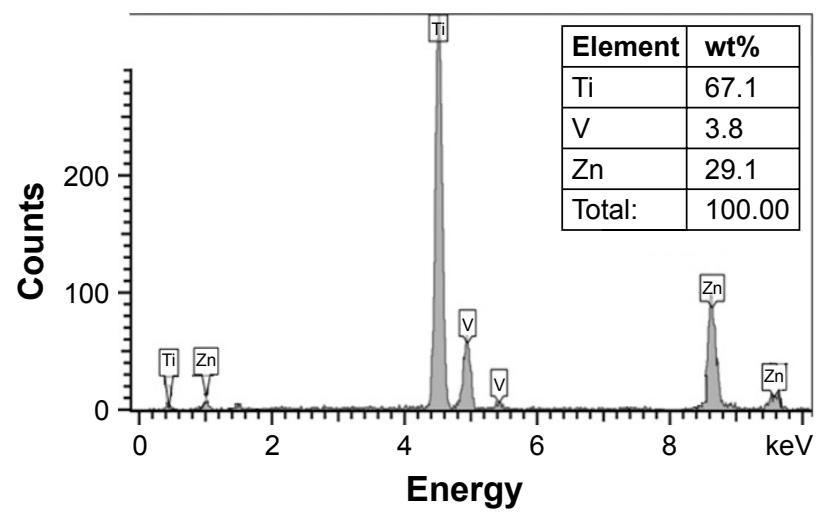

group alloy. Nanocrystals formed during FSP in the upper region (Figure 2A). The lower region, as shown in Figure 2A, has a completely different microstructural morphology and corresponding selected area electron diffraction (SAED) (Figure 2A, inset a2), which indicates that the amorphous phase transformation occurs in this area. Figure 2B shows the $\mathrm{TiZn}_{2}$ phase, and the corresponding SAED also shows discontinuous rings, suggesting the coexistence of both grains and subgrains, as clearly shown in the inset of Figure 2B. As shown in Figure 2C, several nanotwins formed during

B

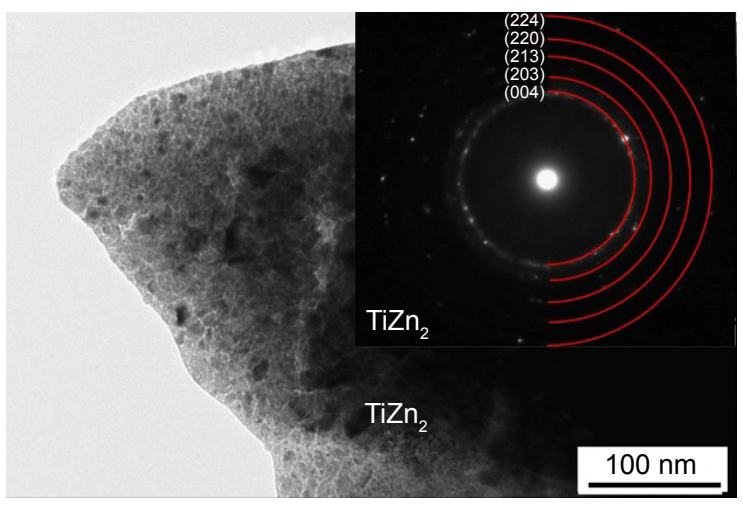

D

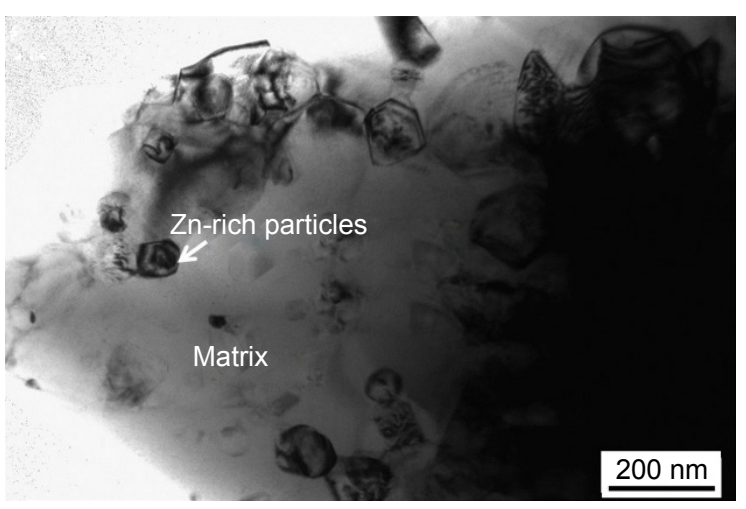

$\mathbf{F}$ Zn-rich particles

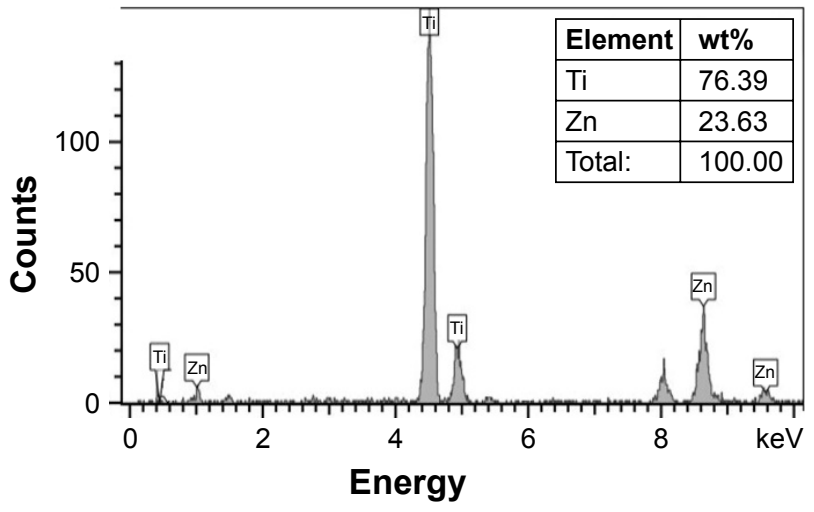

Figure 2 TEM images of upper surface (within $200 \mu \mathrm{m}$ ) in Zn-I-FSP group alloy.

Notes: (A) Nanocrystalline (al) and amorphous (a2), (B) $\mathrm{TiZn}_{2}$, (C) twins, (D) Zn-rich particles and matrix, and (E and F) EDS composition analysis of Zn-rich particles and matrix, respectively, in Figure ID.

Abbreviations: EDS, energy-dispersive X-ray spectroscopy; FSP, friction stir processing; TEM, transmission electron microscopy; wt, weight; Zn, zinc. 

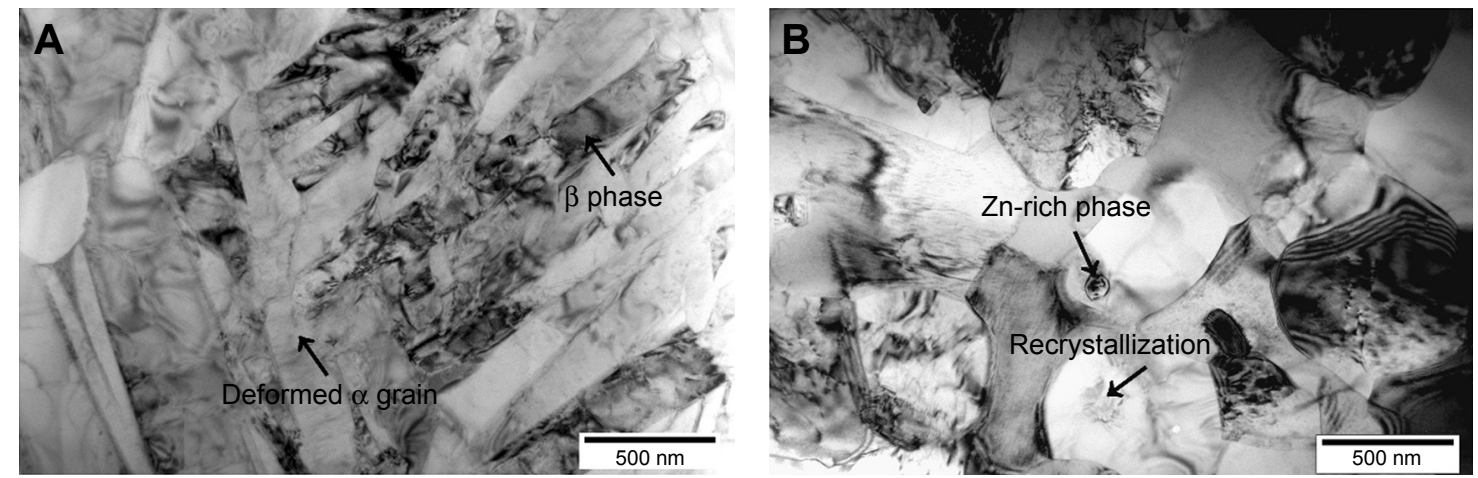

Figure 3 TEM images of middle region ( I mm from upper surface) of stir zone in Zn-I-FSP group alloy.

Notes: (A) Deformed grain and (B) recrystallization grain.

Abbreviations: FSP, friction stir processing; TEM, transmission electron microscopy; Zn, zinc.

FSP in the upper surface of the sample. Several high-density dislocation areas were also observed (not shown). In addition, some Zn-rich particles appeared, as shown in Figure 2D, and the energy-dispersive X-ray spectroscopy (EDS) composition analysis of the matrix revealed that the matrix alloy also contained $\mathrm{Zn}$ (Figure 2E and F), suggesting that during FSP, the added $\mathrm{Zn}$ can transform into a second phase or dissolve into the matrix crystal structure to form a solid solution.

Figure 3 shows the TEM images of middle region of the SZ ( $\sim 1 \mathrm{~mm}$ from upper surface) in the Zn-I-FSP group alloy. Figure $3 \mathrm{~A}$ clearly exhibits deformed and rod-like $\alpha$ grains, suggesting that severe plastic deformation occurs in this area during FSP and that the grains are elongated and refined. Figure 3B shows that new recrystallization is also formed during FSP, which indicates that dynamic recrystallization occurred during FSP, thus forming new grains. This recrystallization occurred because the alloy was simultaneously subjected to severe plastic deformation and the high temperature produced by the stirring effect of the FSP tool. Moreover, grains of the TC4 alloy are fractured or elongated. Meanwhile, grains may have nucleated from areas with high amounts of stored energy, which then grow to form new grains. ${ }^{33}$

The contact angles of water droplets on the surfaces were $61.23^{\circ}$ (Con), $42.43^{\circ}$ (FSP), $30.5^{\circ}$ (Zn-I-FSP), and $39.13^{\circ}$ (Zn-II-FSP) (Figure 4). Thus, the surface of Zn-I-FSP showed the highest hydrophilicity and surface wettability with the addition of $\mathrm{Zn}$ particles. As mentioned earlier, the volumetric ratio of $\mathrm{Zn}$ affected the evolution of microstructural characteristics, which explains the different contact angles of Zn-I-FSP and Zn-II-FSP. Grain refinement seems to be another contributing factor, because the contact angle was smaller for the FSP group than the Con. The difference in contact angle is attributable to the sub-micron/nanoscale grain size and different surface microstructures induced by the different volumetric ratios of added $\mathrm{Zn} .^{22,34}$

\section{Surface properties of TC4/Zn MMNC}

Figure 5 illustrates the force-displacement plots from the nanoindentation tests in each group. The elastic modulus of the Con is $126.23 \pm 19.48 \mathrm{GPa}$. After FSP, the elastic modulus of the FSP group (116.86 $\pm 16.61 \mathrm{GPa})$ decreased and that of the TC4/Zn MMNC surface further decreased with the addition of $\mathrm{Zn}$ particles. The elastic moduli of the Zn-I-FSP and Zn-II-FSP groups are $106.93 \pm 18.99$ and $107.21 \pm 22.43 \mathrm{GPa}$, respectively (Table 1).

The FSP group ( $395.5 \pm 29.44 \mathrm{HV})$ showed the highest microhardness value among the four groups (Table 1). Compared with the Con $(344.6 \pm 15.52 \mathrm{HV})$, the microhardness of the Zn-I-FSP group $(375.7 \pm 13.00 \mathrm{HV})$ was $9.02 \%$ higher and that of the Zn-II-FSP group (389.3 $\pm 26.81 \mathrm{HV})$ was $12.97 \%$

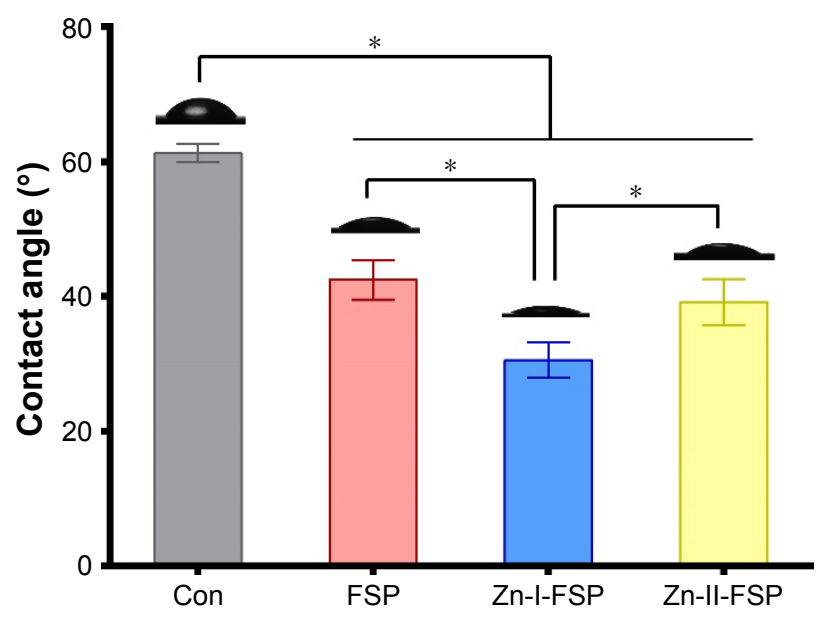

Figure 4 Contact angle of each group.

Notes: Smaller values indicate better hydrophilicity and surface wettability. The surface of Zn-I-FSP exhibited the highest hydrophilicity and surface wettability; $* P<0.05$ compared with the Con.

Abbreviations: Con, control group; FSP, friction stir processing; Zn, zinc. 

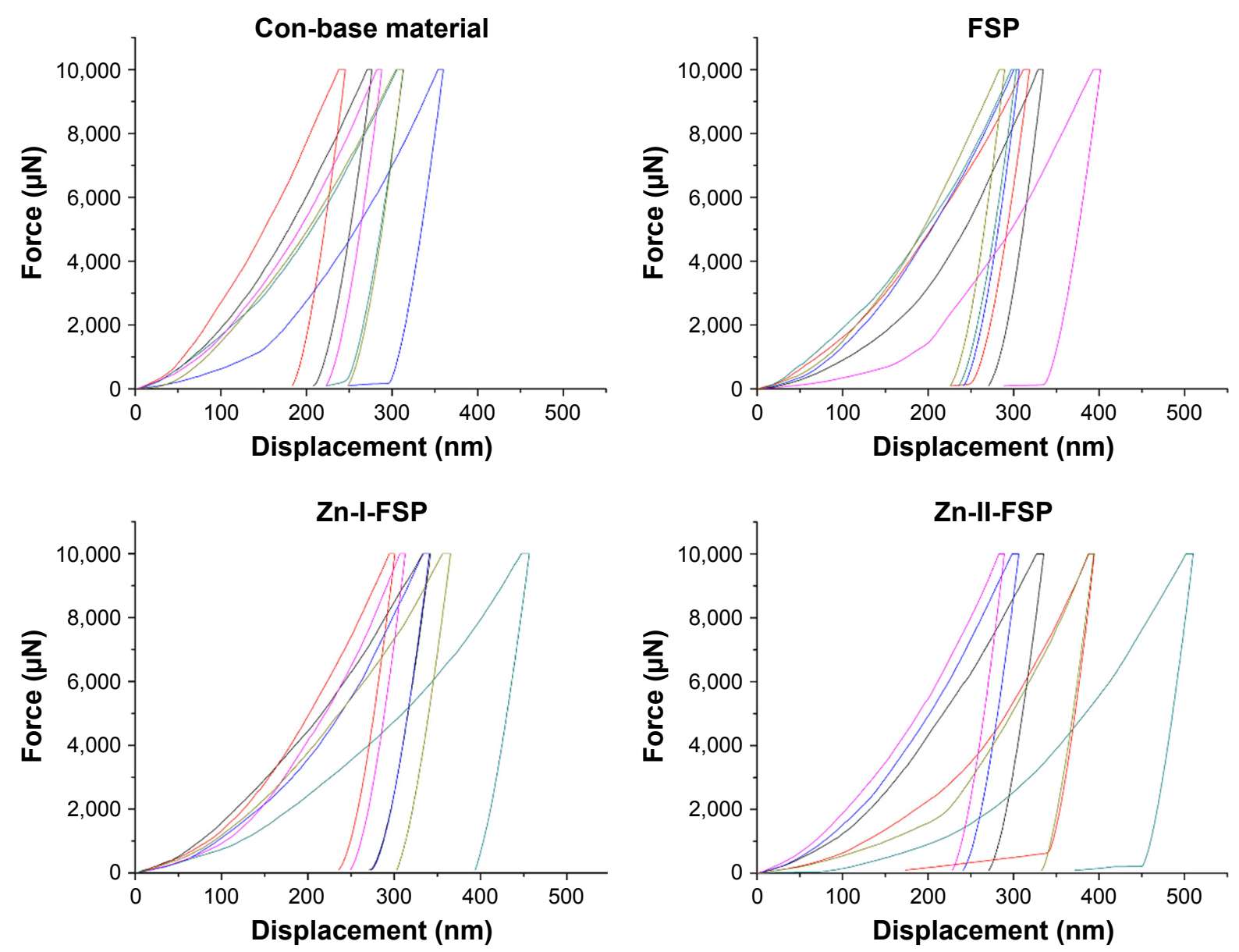

Figure 5 Nanoindentation experiments conducted with Berkovich indent showing force-displacement plots of nanoindentation tests for the each group in stir zone. Note: Different lines represent independent loadings.

Abbreviations: Con, control group; FSP, friction stir processing; Zn, zinc.

higher. The addition of $\mathrm{Zn}$ particles lowered the effect of FSP; however, the increased addition of $\mathrm{Zn}$ decreased the microhardness.

\section{TC4/Zn MMNC promotes cell adhesion and proliferation}

Figure 6A and B shows the adhesion ability of rat BMSCs on the surface of each sample at 1,4 , and $24 \mathrm{~h}$. More cells adhered to the Zn-I-FSP and Zn-II-FSP samples than to the Con samples after 4 and $24 \mathrm{~h}$ (Figure S1). The quantitative

Table I Mechanical properties of each group

\begin{tabular}{lll}
\hline Group & Elastic modulus (GPa) & Microhardness (HV) \\
\hline Con & $126.23 \pm 19.48$ & $344.6 \pm 15.52$ \\
FSP & $116.86 \pm 16.61$ & $395.5 \pm 29.44$ \\
Zn-I-FSP & $106.93 \pm 18.99$ & $375.7 \pm 13.00^{*}$ \\
Zn-II-FSP & $107.21 \pm 22.43$ & $389.3 \pm 26.8 I^{*}$
\end{tabular}

Notes: Data are presented as mean \pm SD. No statistical differences in elastic modulus were found among the groups. *The microhardness showed significant differences among the groups $(P<0.05$ compared with the Con).

Abbreviations: Con, control group; FSP, friction stir processing; Zn, zinc. data further demonstrate that cell adhesion was significantly enhanced on the surface of Zn-I-FSP and Zn-II-FSP groups (Figure 6A). Cell adhesion on surfaces after $24 \mathrm{~h}$ was ranked in the following decreasing order: Zn-I-FSP $>$ Zn-IIFSP $>$ FSP $>$ Con.

To further confirm the expression of an adhesion-related protein and to observe initial cell spreading, integrin $\beta 1$ and the cytoskeleton of BMSCs in each group were examined by immunofluorescence after staining with a FITC-tagged antibody and TRITC-phalloidin to visualize integrin $\beta 1$ and cytoskeletal actin expression, respectively (Figure 6B). The nuclei of BMSCs were stained with DAPI. The BMSCs on each sample exhibited a multipolar spindle-like morphology and extended pseudopodia on all surfaces. The cells seeded on the Zn-I-FSP surface expressed the highest level of integrin $\beta 1$, and those on the FSP and Zn-II-FSP group expressed higher levels of integrin $\beta 1$ than those on the Con.

The MTT results are shown in Figure 6C. No statistically significant differences were observed among the four groups on day 1 . On day 4 , there was no significant difference in the 
A Cell adhesion

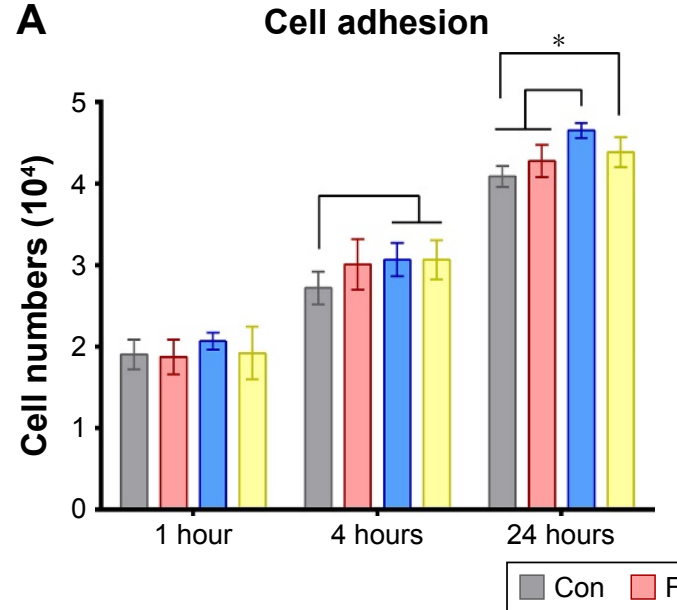

C
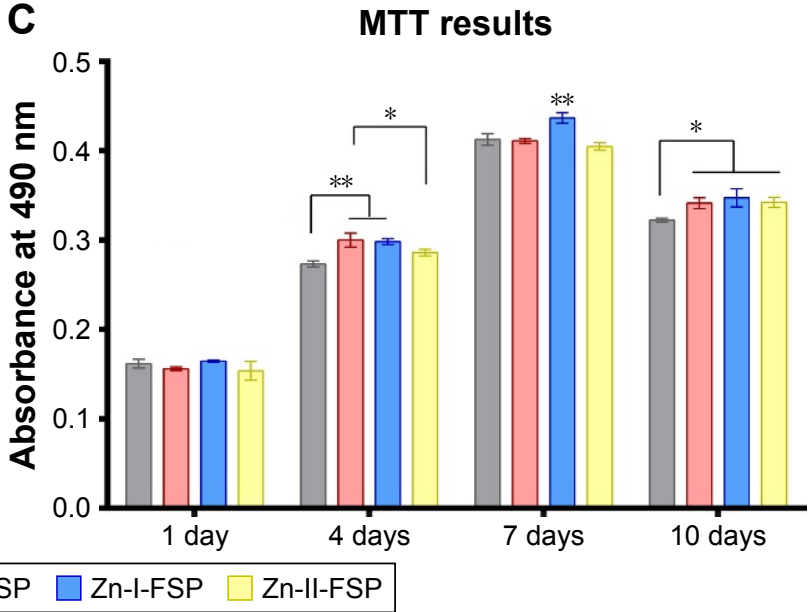

B
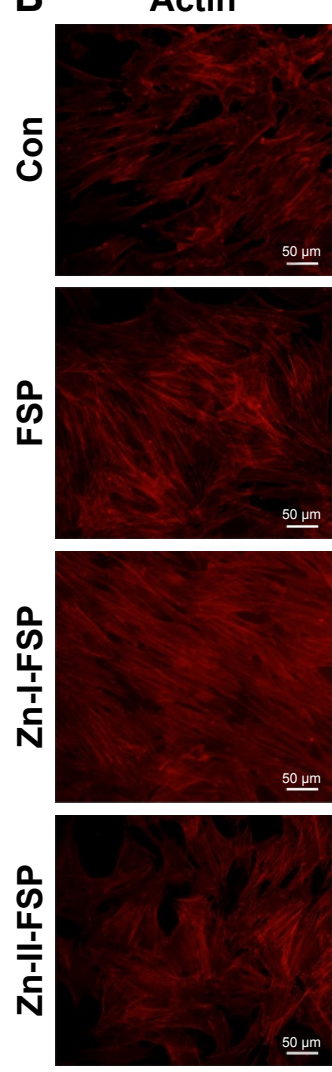

Integrin $\beta 1$
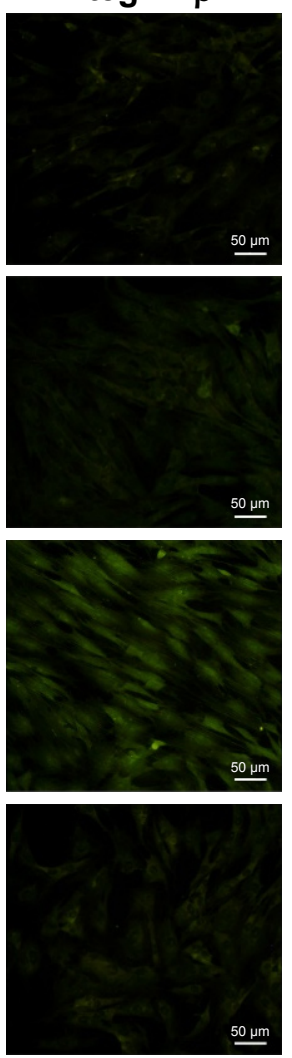
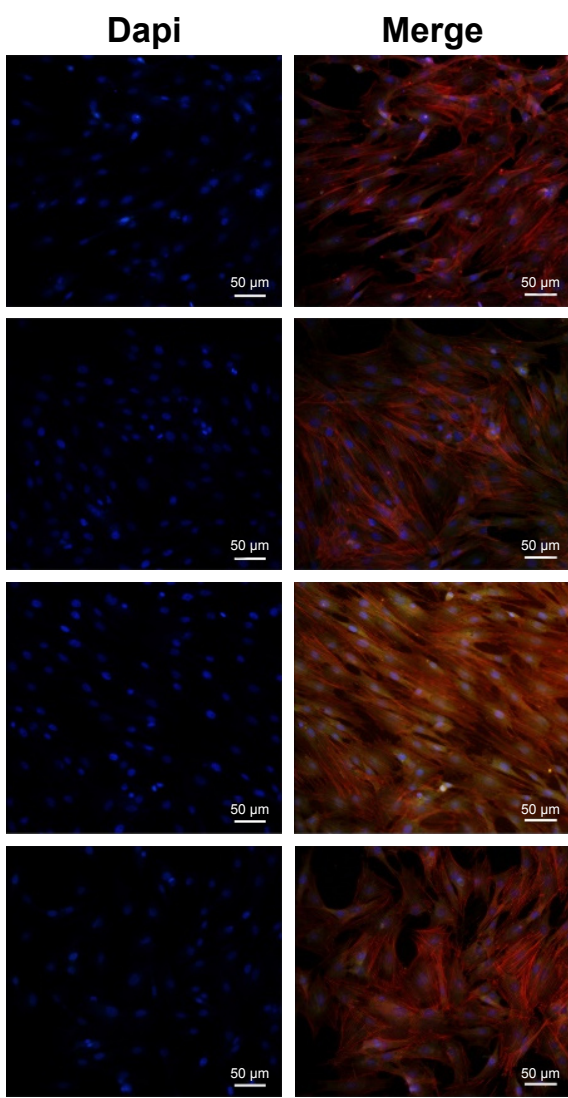
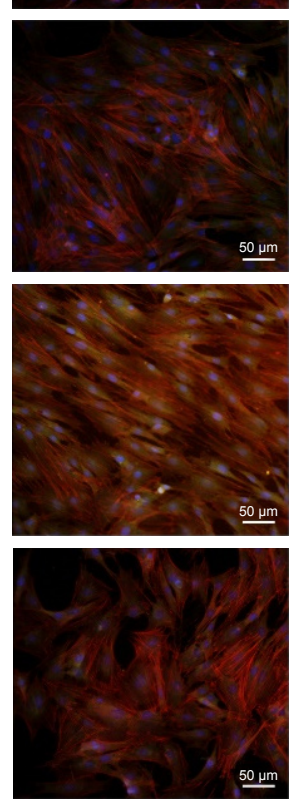

Zoom
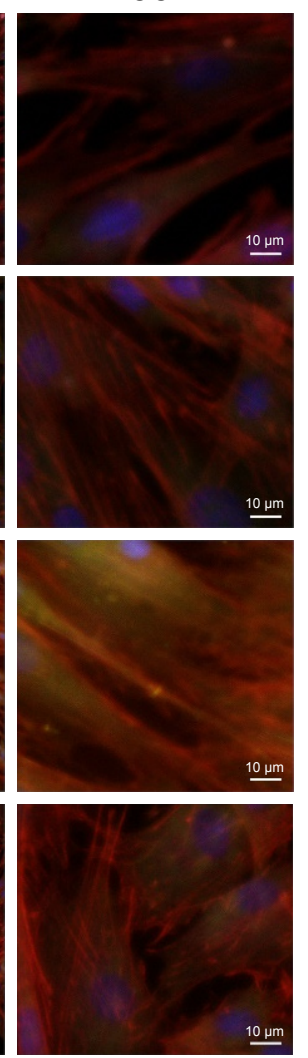

Figure 6 Cell adhesion and proliferation analysis.

Notes: (A) Cell counts for each group at I, 4, and $24 \mathrm{~h} ; * P<0.05$. (B) Detection of integrin $\beta \mathrm{I}$ immunofluorescence and cell spreading after $24 \mathrm{~h}$ of culture. Red represents the actin cytoskeleton of BMSCs; green represents integrin $\beta$ I expression in BMSCs; and blue represents the nuclei of BMSCs stained with DAPI. (C) MTT assay results. Metabolic activity levels of BMSCs after I, 4, 7, and 10 days of culture, as determined by MTT assay; $* P<0.05, * * P<0.01$.

Abbreviations: Con, control group; BMSCs, bone marrow stromal cells; FSP, friction stir processing; Zn, zinc.

total cell metabolic activity between the FSP and Zn-I-FSP groups; however, that of both groups was higher than that of the Con. On day 7, the total cell metabolic activity in the Zn-I-FSP group was significantly greater than that in the other groups. On day 10, all three modified groups showed more stimulated total cell metabolic activity than the Con. The MTT results indicate that the novel surface nanocomposite positively affects the viability and proliferation of the BMSCs.

\section{TC4/Zn MMNC enhances in vitro osteogenic differentiation}

As shown in Figure 7A, all groups showed intense ALP staining on days 7 and 14 of culture, except for the Con, which showed light staining. Quantitative ALP activity assay of the Zn-I-FSP group on day 7 showed significantly higher activity than that in the Con, whereas BMSCs in the Zn-II-FSP group showed higher ALP activity on day 

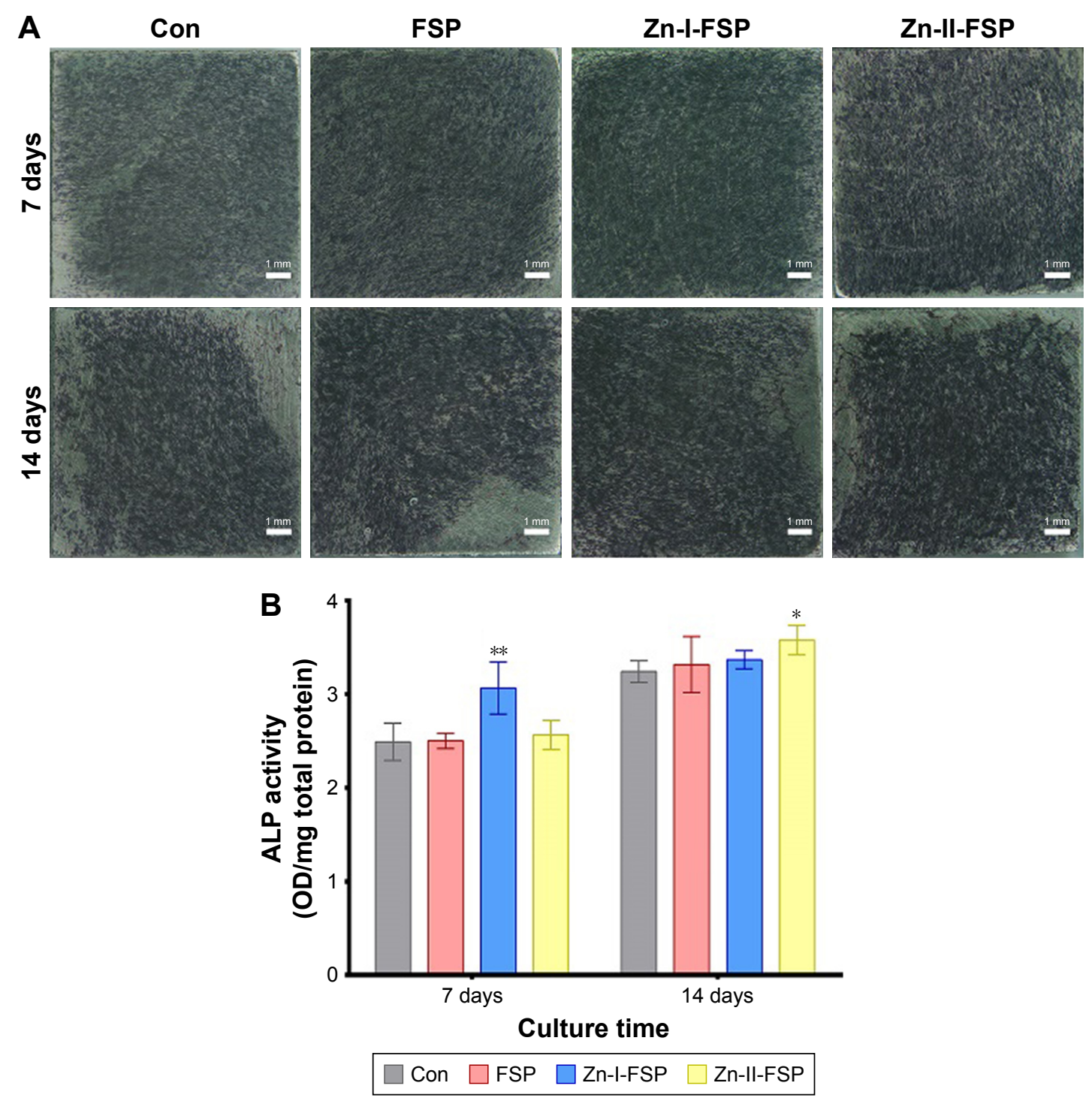

Figure 7 The ALP activity results.

Notes: (A) ALP staining of BMSCs in each sample on days 7 and 14 of culture. (B) ALP activity of BMSCs in each sample, as determined by p-nitrophenyl phosphate assay on days 7 and 14 of culture; $* P<0.05$, $* * P<0.01$.

Abbreviations: ALP, alkaline phosphatase; BMSCs, bone marrow stromal cells; Con, control group; FSP, friction stir processing; OD, optical density; Zn, zinc.

14 than those in the Con. The ALP activity results indicate enhanced osteogenic differentiation of BMSCs seeded on the TC4/Zn MMNC surface with the addition of $\mathrm{Zn}$ particles (Figure 7B).

The expression levels of osteogenic differentiation marker genes, including Runx2, ALP, OSX, COL- $1 \alpha$, OPN, and OCN, were examined by PCR after 7- and 14-day cultures. As shown in Figure 8, after a 7-day culture, the expression of Runx2, ALP, COL-1 $\alpha$, and OPN was higher in the Zn-I-FSP and Zn-II-FSP groups than that in the Con, whereas Runx 2 and COL- $1 \alpha$ showed higher expression in the FSP group than in the Con. The highest expression of COL-1 $\alpha$ was observed in the FSP group. The expression of COL-1 $\alpha$ was ranked in the order of FSP $>$ Zn-II-
FSP $>$ Zn-I-FSP $>$ Con. After a 14-day culture, the expression of Runx2, OPN, and OCN in the Zn-II-FSP group was significantly upregulated compared with expression in the other three groups. The expression of ALP in the Con was lower than that in the other three groups. The expression of COL- $1 \alpha$ was downregulated in the experimental groups compared with expression in the Con after culturing for 14 days. Compared with the Con, the Zn-I-FSP group showed upregulated OCN expression.

\section{TC4/Zn MMNC promotes rapid osseointegration}

An in vivo study was performed to further detect the osseointegration of the TC4/Zn MMNC implant. Figure 9A 
Runx2
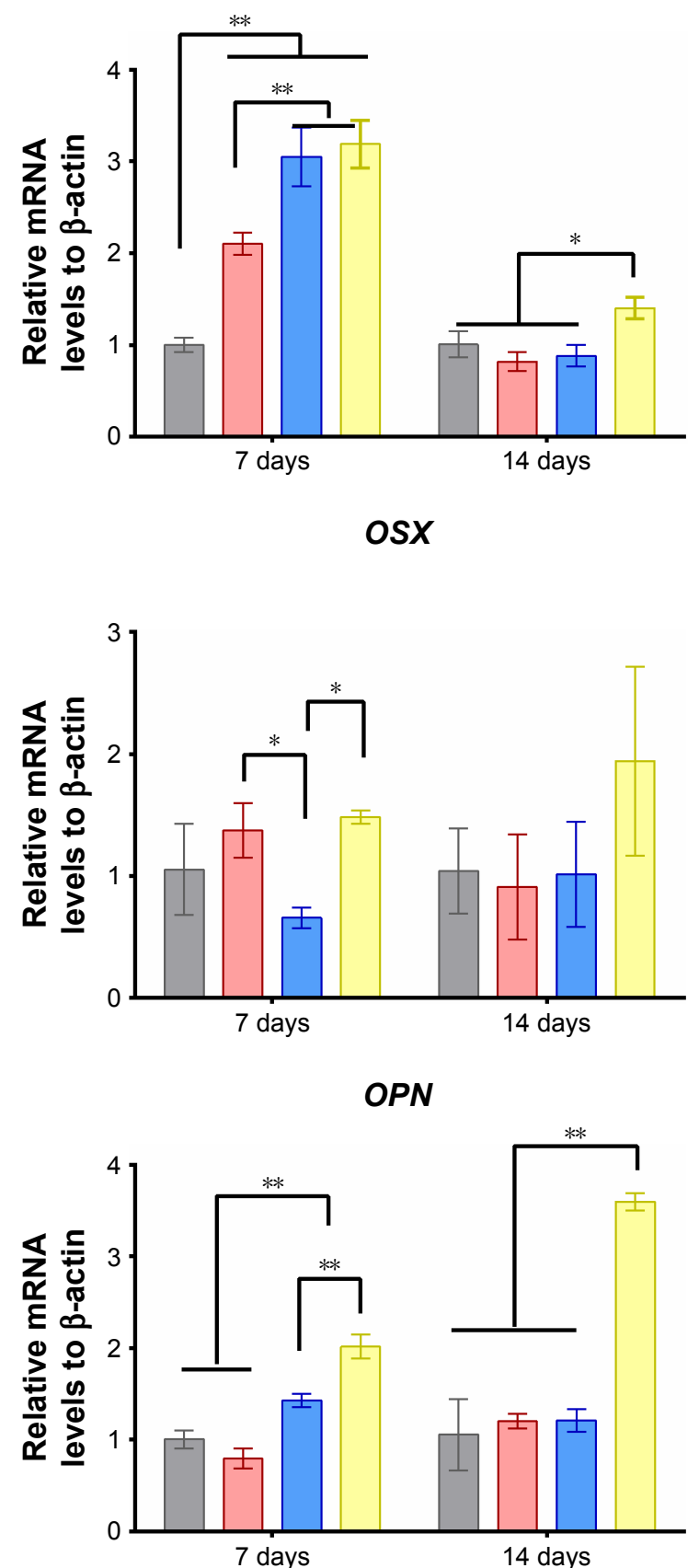

$\square$ Con $\square$ FSP
ALP
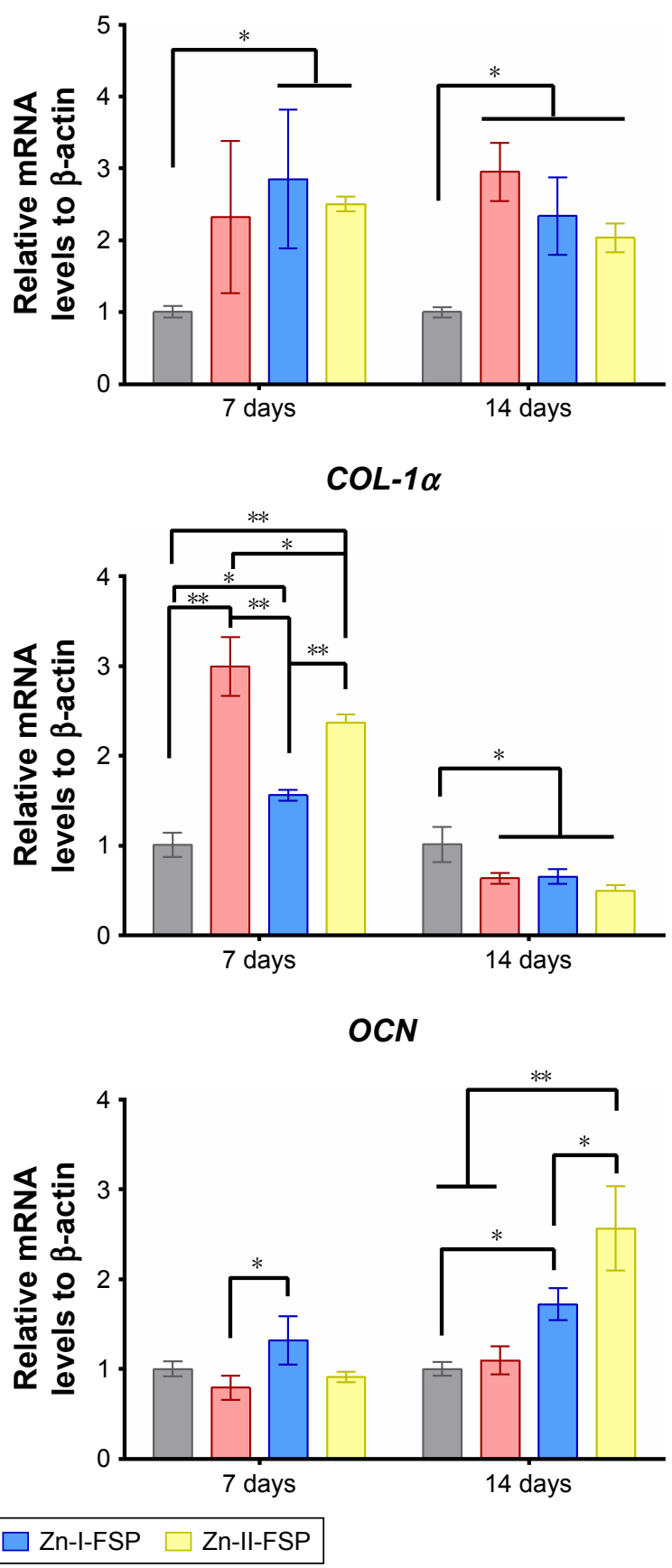

Figure 8 Expression levels of osteogenic differentiation marker genes.

Notes: Gene expression of Runx2, ALP, OSX, COL-I $\alpha, O P N$, and OCN genes on days 7 and 14 assayed by real-time $P C R ; * P<0.05$, $* * P<0.01$.

Abbreviations: ALP, alkaline phosphatase; Con, control group; FSP, friction stir processing; Zn, zinc.

shows images of the intersecting surface of the implant captured by micro-CT. The new bone around the implant surface appeared to have a high-density structure, and this structure was larger round the implant surfaces in the marrow cavity of the FSP, Zn-I-FSP, and Zn-II-FSP samples than in that of the Con samples. The BV, BV/TV, and Tb.N around the implant (within $2 \mathrm{~mm}$ from the implant) were determined
(Figure 9B-D). The Zn-II-FSP group demonstrated a higher $\mathrm{BV}$ than that of the other groups. In terms of the Tb.N, the Zn-I-FSP group exhibited obvious advantages among the four groups. Both the FSP and Zn-II-FSP groups exhibited a higher BV/TV than the other two groups.

The sequential fluorescent labeling analysis results are shown in Figures 10 and 11. After 2 weeks, the percentage of 


\section{A}
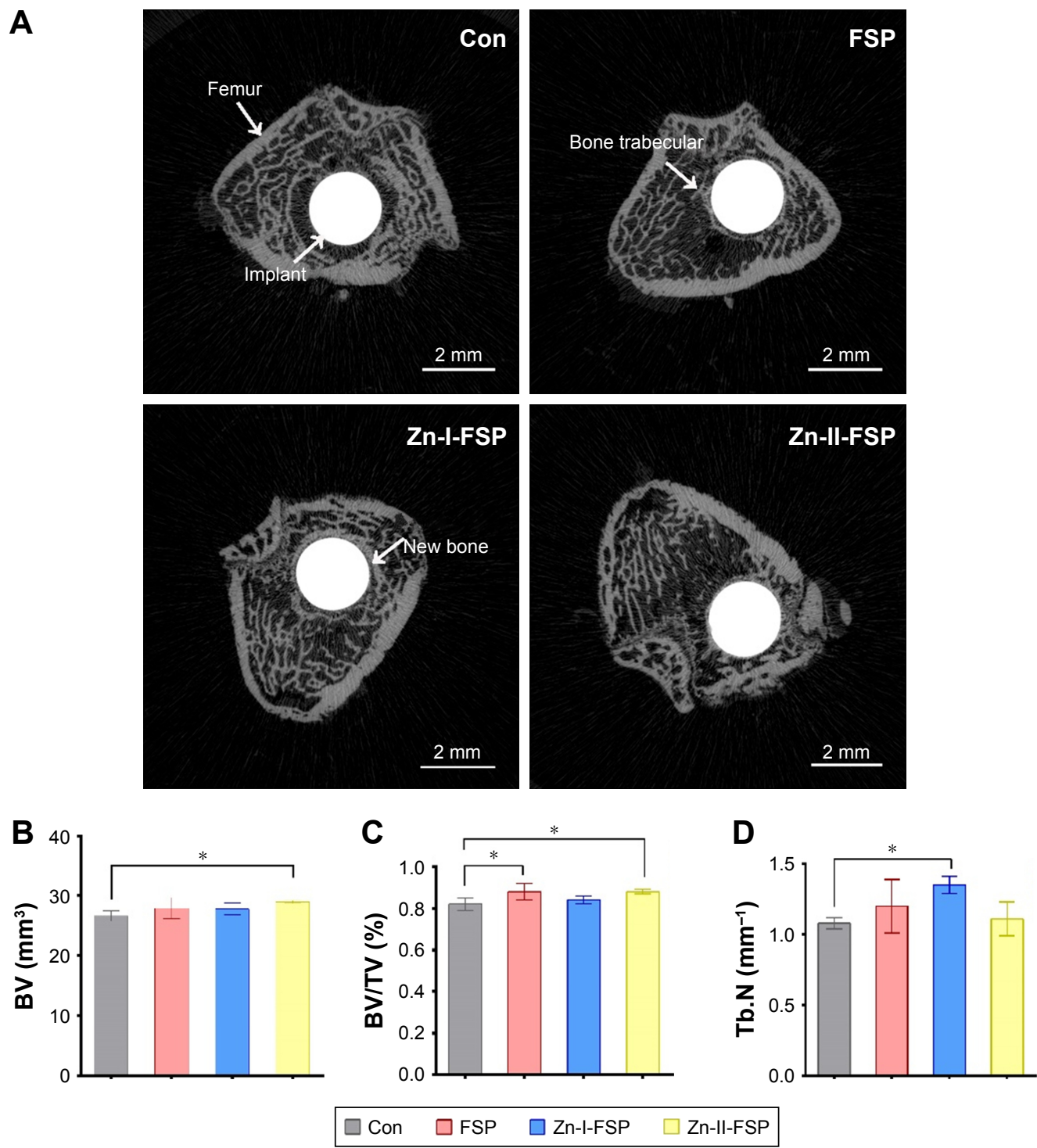

Figure 9 Micro-CT analysis of each group.

Notes: (A) Micro-CT images of femurs 8 weeks after implantation. New bone with a high-density structure was observed around the implant. (B-D) Analysis of the BV, BV/TV, and Tb.N around the implant (within $2 \mathrm{~mm}$ from the implant); $* P<0.05$.

Abbreviations: BV, bone volume; micro-CT, microcomputed tomography; Con, control group; FSP, friction stir processing; Tb.N, trabecular number; TV, total volume; Zn, zinc.

TE labeling area in the Zn-I-FSP group was found to be the largest among the four groups and the labeling area of the Con was significantly lower than that of the other groups. After 4 weeks, the percentage of AL labeling in the Zn-II-FSP group was obviously larger than that in the other groups. At the last time point, the percentage of CC labeling area in the Zn-I-FSP group and Zn-II-FSP group was larger than that in the other two groups. The sequential fluorescent labeling results indicate that the TC4/Zn MMNC stimulates more new bone formation at every time point, especially in the form of Zn-II-FSP.

Figure 12A shows the position of the implant in the distal femur of the SD rats. Histological analyses are shown in Figure 12B. The partial magnifications of histological stain reveal the formation of fibrous connective tissue around the Con and FSP implants, which separated the new bone tissue from the implant surface (marked by red arrows). The BIC was calculated as the quotient of the length of the direct BIC and the length of the implant. The BIC index of Zn-I-FSP and Zn-II-FSP was $56.0 \% \pm$ $7.26 \%$ and $58.5 \% \pm 5.45 \%$ (Figure 12C), respectively, both of which are significantly larger than that of Con $(35.25 \% \pm 6.24 \%)$ and FSP $(42.75 \% \pm 4.65 \%)$, suggesting that the two TC4/Zn MMNC groups induced better BIC among the four groups. 

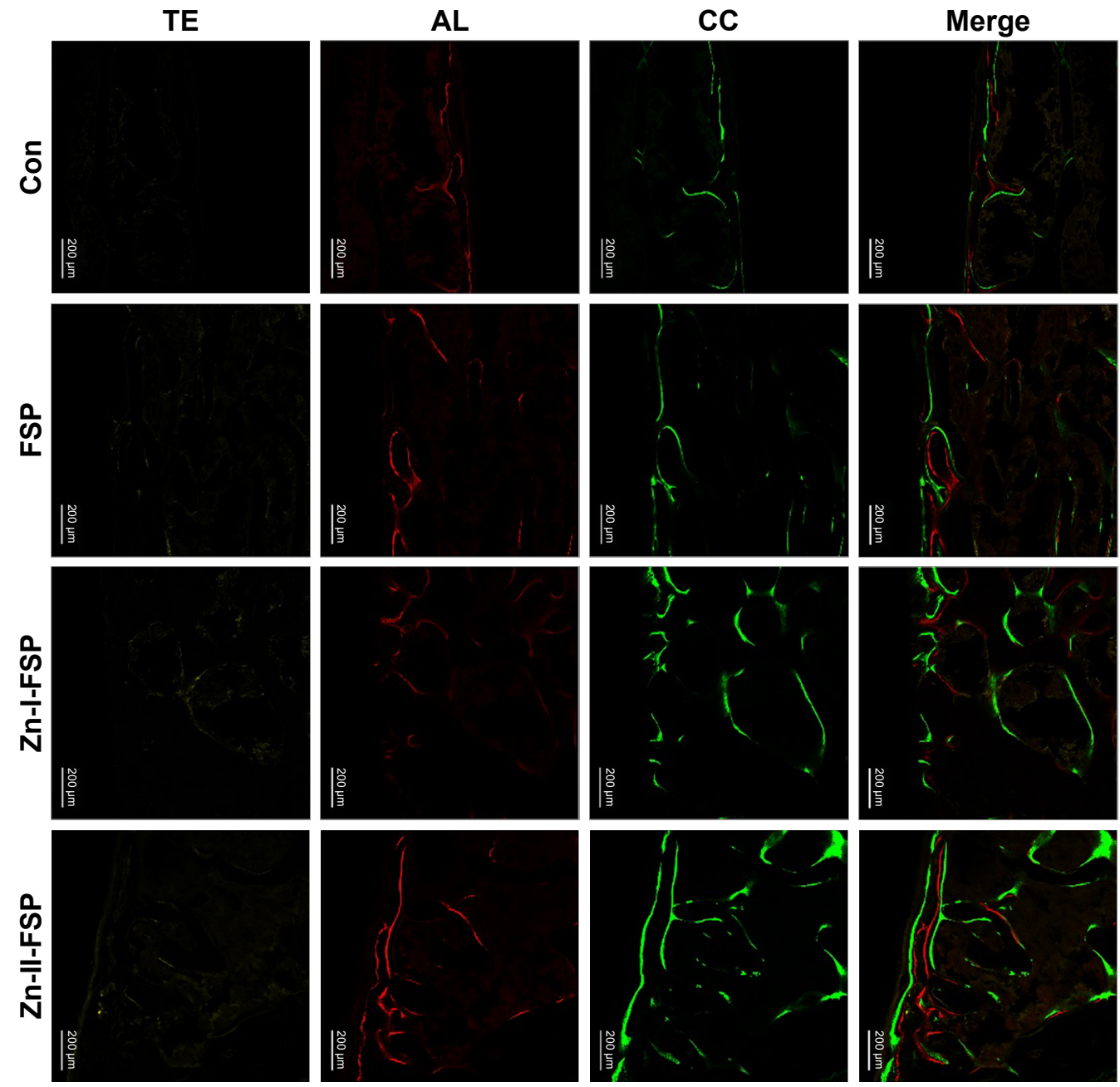

Figure 10 Sequential fluorescent labeling observations of TE, AL, and CC at 8 weeks after implantation.

Notes: The color in yellow (TE), red (AL), and green (CC) indicates the rate of new bone formation at 2, 4, and 6 weeks after the operation, respectively. Scale bar: $200 \mu \mathrm{m}$. Abbreviations: AL, alizarin red S; CC, calcein; Con, control group; FSP, friction stir processing; TE, tetracycline; Zn, zinc.

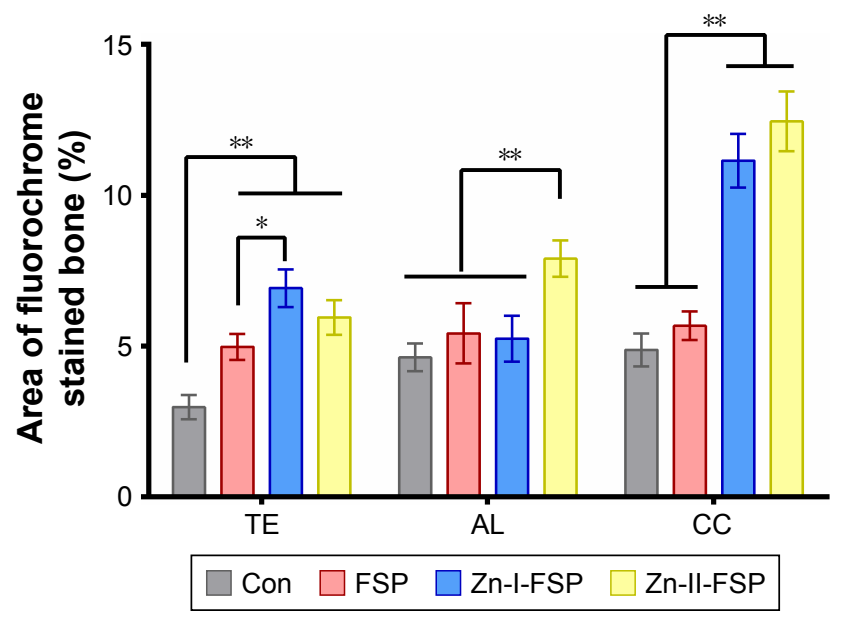

Figure II The area percent of fluorochrome stained bone in each group.

Notes: The TE, AL, and CC indicated the rate of new bone formation at the 2, 4, and 6 weeks after the operation, respectively; $* P<0.05$, $* * P<0.01$ ).

Abbreviations: $\mathrm{AL}$, alizarin red S; CC, calcein; Con, control group; FSP, friction stir processing; TE, tetracycline; Zn, zinc.

\section{Discussion}

Microstructural evolution and mechanical properties of TC4/Zn MMNC

Microstructural observations indicate that the TC4 alloy undergoes intense plastic deformation at the elevated temperature during FSP and that the peak temperature exceeds the phase transformation point..$^{33}$ Thus, the microstructures transform completely to the $\beta$ phase. During cooling, the $\alpha$ phase preferentially nucleates at triple junctions and boundaries of $\beta$ grains with a low energy orientation relationship, leading to the formation of a GB $\alpha$ phase (Figure 1D, F, and $\mathrm{H}$ ). Thereafter, the acicular $\alpha$ and martensite $\alpha^{\prime}$ phases form in the interior of $\beta$ grains. Martensite $\alpha^{\prime}$ can form when the TC4 alloy is cooled from above the $\beta$ transition temperature at a cooling rate of $>20^{\circ} \mathrm{C} / \mathrm{s} .^{35}$ Therefore, we believe that the finer microstructures formed during FSP are 
A

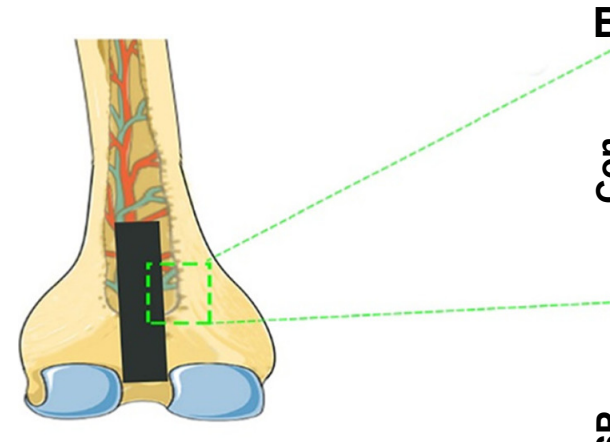

\section{C}

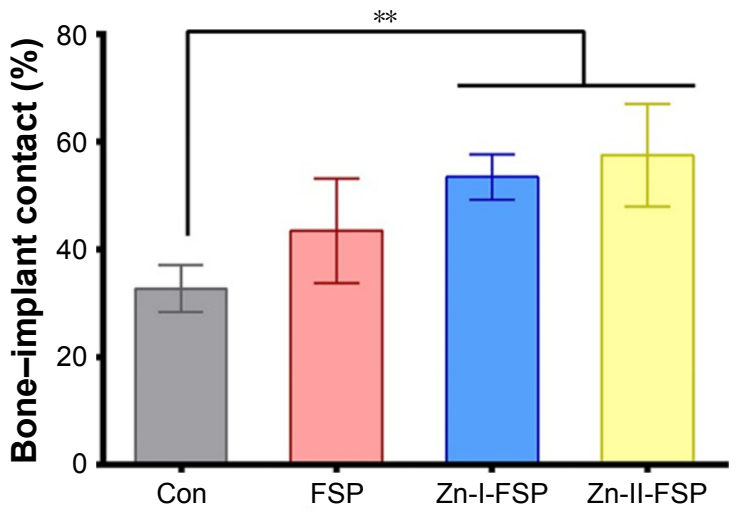

B
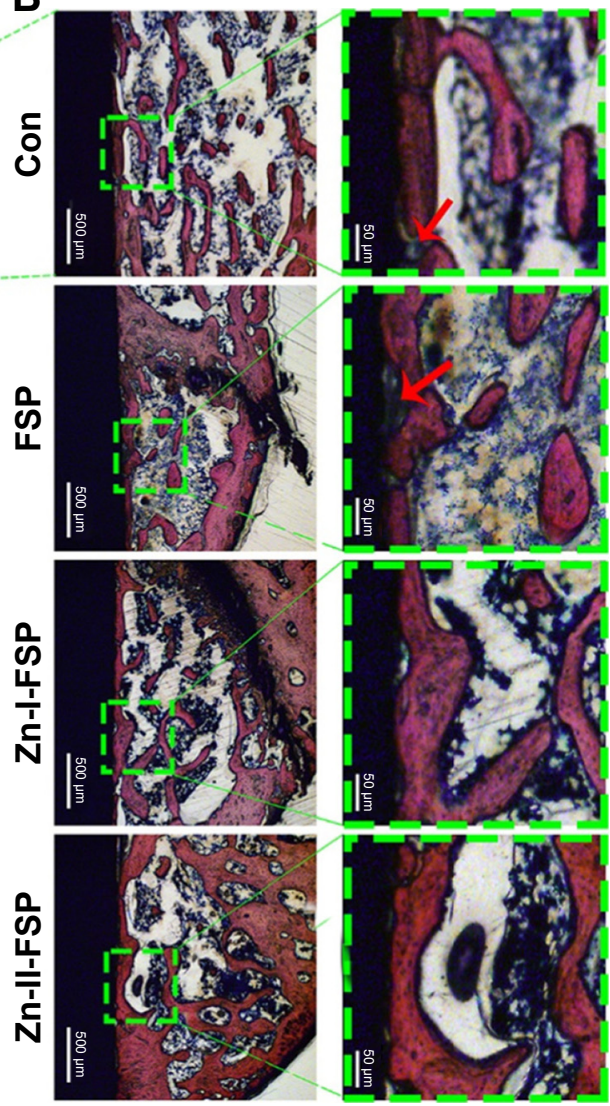

Figure 12 The analysis of histological images and BIC.

Notes: (A) The position of the implant in the distal femur. (B) Histological images of each group and partial magnifications (green squares) at 8 weeks after implantation. Red arrows: fibrous connective tissue. (C) BIC results from the histomorphometric measurements; ${ }^{* * P}<0.0 \mathrm{I}$.

Abbreviations: BIC, bone-implant contact; Con, control group; FSP, friction stir processing; Zn, zinc.

the main reason for the higher microhardness of the FSPed TC4 alloy. This result is consistent with those of Li et al's? investigation, in which the greater surface microhardness of the TC4 alloy after FSP was attributed to finer $\alpha / \beta$ lamella. These researchers also concluded that the martensite $\alpha^{\prime}$ phase could enhance the hardness of the FSPed TC4 alloy. ${ }^{7}$ In addition, especially on the upper surface of the FSPed samples, nanocrystalline, amorphous, nanotwins, highdensity dislocations, among other features, form during FSP due to more severe plastic deformation (Figure 2A, C, and E). These microstructural features can significantly increase the microhardness of the TC4 alloy, which has been reported in many investigations. ${ }^{36-40}$ For example, Liu et a ${ }^{36,37}$ considered that the nanocrystallinity and high-density dislocations of the TC4 alloy formed via shot peening could result in remarkable grain refinement strengthening and work-hardening of the alloy, which would significantly improve their surface microhardness. The amorphous metal has no dislocations or GBs, resulting in higher microhardness, showing extraordinary yield strength and microhardness..$^{38-40}$
After adding $\mathrm{Zn}$ into the TC4 alloy, $\mathrm{Zn}$ can transform into a second phase or dissolve into the matrix crystal structure to form a solid solution (Figure 2B, D, and F). However, the addition of $\mathrm{Zn}$ into the TC4 alloy will also result in an increase in the size of the $\beta$ phase region (Figure 1E and G), which may explain why the FSP-Zn group has a lower microhardness than the FSP group and why the microhardness decreases with the increase in the $\mathrm{Zn}$ content (Table 1). However, the reason that elemental $\mathrm{Zn}$ can accelerate the growth of $\beta$ grains is not clear and requires further investigation.

The nanoindentation test results showed that the FSP group and FSP/Zn groups have lower elastic moduli than the Con, which can be attributed to the relatively lower $\beta$ phase fraction in the TC4 alloy due to the higher content of $\alpha$ phase-stabilizing element Al. After FSP, more $\beta$ phase forms due to the faster cooling rate (Figure 1D, F, and H), while the Ti alloy with the higher $\beta$ phase content has a lower elastic modulus. ${ }^{16,17,41}$ On the other hand, the MMNC produced by FSP has very small grains and, in our previous study, the material with more refined grains exhibited 
a lower elastic modulus. ${ }^{18}$ Therefore, the FSP and MMNC groups have lower elastic moduli than the Con. After adding $\mathrm{Zn}$ into the TC4 alloy, although the size of the $\beta$ region increased, more $\beta$ phase may simultaneously form, which may be why FSP/Zn MMNC has a lower elastic modulus than the FSP group.

In medical implants, metal ion release from the Ti alloy inevitably occurs due to wear or corrosion ${ }^{1}$ and this release may cause an immune response, such as inflammation around or an allergy to the implant. The wear properties of the material are positively correlated with hardness according to the Archard principle. ${ }^{42,43}$ Through grain refinement and the change in the $\alpha / \beta$ ratio caused by FSP, the microhardness of the Zn-I-FSP group was significantly increased by $9.02 \%$ and that of the Zn-II-FSP group was increased by $12.97 \%$. These changes indirectly indicate that fewer metal ions would be released. In terms of corrosion, human body fluids are complex electrolyte media that contain a variety of organic acids, oxides, and ions. Corrosion reactions of medical implants will occur inevitably over the long term. ${ }^{8}$ Multiple studies have reported that nanocomposites modified by FSP have enhanced corrosion resistance. ${ }^{19,20}$ In the corrosive environment of a sodium chloride solution, the electronegativity of $\mathrm{Zn}$ is stronger than that of $\mathrm{Al}$, meaning that $\mathrm{Zn}$ is more likely to be released. Nevertheless, further investigations are required to evaluate the corrosion resistance of this novel material.

The improved surface wettability of the material contributed to the different cell adhesion and osteogenic responses observed. The surface properties of a biomaterial directly affect early cell adhesion. In this study, the improved surface wettability is thought to contribute to the high numbers of cells adhering to the surface of Zn-I-FSP and Zn-II-FSP. Cellular adhesion is the primary stage of the interaction between the tissue and the medical implants, followed by cell proliferation and differentiation. A series of specific proteins are thought to be involved in signaling transduction during cell adhesion. ${ }^{44}$ Integrin, as an extracellular matrix receptor, participates in the signal transduction involved in responding to external stimuli, regulating gene expression, and influencing cell migration, proliferation, and differentiation. ${ }^{45}$ The detection of the expression of integrin $\beta 1$ confirmed the crucial role of this adhesion-related protein in the process of cell adhesion. Surface wettability has been reported to be an important factor for controlling the enhanced attachment and spreading of stem cells. ${ }^{22,23}$ However, further investigations are required to elucidate the exact mechanism.
The surface nanocomposite modified by FSP promoted the proliferation of BMSCs, as previously reported. ${ }^{18}$ ALP is an early marker of osteogenesis, and its upregulation indicates enhanced phosphate metabolism. In this study, the ALP upregulation in Zn-I-FSP and Zn-II-FSP groups indicated positive regulation via $\mathrm{Zn}$ release. $\mathrm{Zn}$ is an important cofactor for ALP; moreover, it can activate Zn-containing metalloenzymes, including collagen enzyme, DNA polymerase, and RNA polymerase, which are directly involved in the expression of nucleic acids and protein that are closely associated with bone metabolism. Multiple studies have shown that $\mathrm{Zn}$ can upregulate the osteogenic differentiation of BMSCs. ${ }^{27,46,47}$ Huo et $\mathrm{al}^{48}$ added $\mathrm{Zn}$ to the coating of a Ti surface using anodization and hydrothermal treatments and achieved enhanced osteogenic activity and antibacterial properties. ERK1/2 signaling was found to be involved in these processes.

The PCR results confirmed the enhanced ALP activity at the gene level. Early enhanced expression of Runx2, a key gene in osteogenesis, can further activate and upregulate the expression of other related genes. ${ }^{49,50}$ OSX accelerates the process of osteogenic differentiation and new bone formation. ${ }^{26}$ In this study, the role of OSX was not obvious. It has been reported that Col-1 $\alpha$ can promote adhesion behavior mediated by integrin $\beta 1 .{ }^{51} \mathrm{Col}-1 \alpha$ exerts a considerable influence on the early deposition of inorganic molecules. OPN is associated with adhesion and matrix synthesis before mineralization and is a relatively late marker. ${ }^{52}$ $\mathrm{OCN}$ is a later marker of osteogenesis. The upregulation of OCN contributes to mineral deposition in the extracellular matrix and new bone mineralization..$^{31}$ The enhanced expression of these osteogenic differentiation marker genes prompted in vitro BMSCs osteogenic differentiation at different stages.

TE, AL, and CC fluorescent markers can integrate with calcium ions in bone tissue and form complexes deposited in situ. The speed of calcium deposition around implants can be recorded using sequential fluorescent labeling to evaluate new bone formation. The enhanced rapid osseointegration of Zn-I-FSP and Zn-II-FSP was closely related to the positive effects of proliferation and osteogenic differentiation of BMSCs, which imbued the local environment with more osteoblasts around the implant.

Hydroxyapatite in natural bone takes the form of acicular nanocrystals $(<100 \mathrm{~nm}){ }^{45}$ The nanocrystalline structure of TC4/Zn MMNC was similar to the crystal structure of hydroxyapatite in natural bone; thus, it could biominmetically promote the growth of hydroxyapatite. In addition, the release 
of $\mathrm{Zn}$ in the body can promote extracellular matrix-related protein secretion and new bone mineralization.

According to the guidelines of classical implant dentistry, before the prosthetic procedure and loading, at least 3 months are required for adequate osseointegration after implant surgery. In this study, rapid osseointegration in vivo of the novel TC4/Zn MMNC surface was enhanced compared with the smooth TC4 surface. However, further research comparing this new implant material with classical modified implants should be conducted. Furthermore, how the nanocrystalline structure of TC4/Zn MMNC elicits responses in rat BMSCs requires further elucidation.

\section{Conclusion}

To strengthen the surface mechanical properties and promote the osteogenic capacity of this biomaterial, we modified the surface of TC4 through FSP with the addition of Zn particles and successfully constructed a TC4/ $\mathrm{Zn}$ MMNC surface with grain refinement. Based on the characterization of the microstructural evolution, the mechanical properties were improved. Nanocrystalline and numerous $\beta$ regions, GB $\alpha$ phase, coarser acicular $\alpha$ phase, and finer acicular martensite $\alpha^{\prime}$ appeared because of the severe plastic deformation caused by FSP, resulting in a decrease in the elastic modulus and an increase in the surface hardness. With the addition of $\mathrm{Zn}$ particles and the enhancement of hydrophilicity, biocompatibility was greatly promoted in terms of cell adhesion and proliferation. The in vitro osteogenic differentiation of rat BMSCs on the novel TC4/Zn MMNC surface and the in vivo osseointegration of the TC4/Zn MMNC implant were enhanced. The TC4/Zn MMNC surface modified using FSP not only exhibited superior mechanical properties but also provided improved surface and physicochemical properties for cell attachment and osseointegration. This novel TC4/Zn MMNC shows great potential for dental and orthopedic applications.

\section{Acknowledgments}

This research was supported by the National Natural Science Foundation of China (project numbers: 81300919, 81300912, 51302168, and 51674167), the Project of Science and Technology Commission of Shanghai Municipality (project number: 12441903002), the Shanghai Pujiang Program (15PJD017), and the SMC-ChengXing Project, Shanghai Jiao Tong University.

\section{Disclosure}

The authors report no conflicts of interest in this work.

\section{References}

1. Rack HJ, Qazi JI. Titanium alloys for biomedical applications. Mater Sci Eng C. 2006;26(8):1269-1277.

2. Granchi D, Cenni E, Trisolino G, Giunti A, Baldini N. Sensitivity to implant materials in patients undergoing total hip replacement. J Biomed Mater Res B Appl Biomater. 2006;77B(2):257-264.

3. Heimann RB. Structure, properties, and biomedical performance of osteoconductive bioceramic coatings. Surf Coat Technol. 2013;233:27-38.

4. Wu AY, Hsu JT, Huang HL. An in vitro biomechanical evaluation of a new commercial titanium-zirconium alloy dental implant: a pilot study. Implant Dent. 2014;23(5):534-538.

5. Long M, Rack HJ. Titanium alloys in total joint replacement - a materials science perspective. Biomaterials. 1998;19(18):1621-1639.

6. Ding Z, Zhang C, Xie L, Zhang LC, Wang L, Lu W. Effects of friction stir processing on the phase transformation and microstructure of TiO2-compounded Ti-6Al-4V alloy. Metall Mater Trans A. 2016; 47(12):5675-5679.

7. Li B, Shen Y, Hu W, Luo L. Surface modification of Ti-6Al-4V alloy via friction-stir processing: microstructure evolution and dry sliding wear performance. Surf Coat Technol. 2014;239:160-170.

8. Asri RIM, Harun WSW, Samykano M, et al. Corrosion and surface modification on biocompatible metals: a review. Mater Sci Eng C. 2017;77: 1261-1274.

9. Zatta PF, Alfrey AC. Aluminium Toxicity in Infants' Health and Disease. World Scientific. Singapore; 1998.

10. Sabbioni E, Kuèera J, Pietra R, Vesterberg O. A critical review on normal concentrations of vanadium in human blood, serum, and urine. Sci Total Environ. 1996;188(1):49-58.

11. Gefen A. Computational simulations of stress shielding and bone resorption around existing and computer-designed orthopaedic screws. Med Biol Eng Comput. 2002;40(3):311.

12. Liu W, Cheng M, Wahafu T, et al. The in vitro and in vivo performance of a strontium-containing coating on the low-modulus $\mathrm{Ti} 35 \mathrm{Nb} 2 \mathrm{Ta} 3 \mathrm{Zr}$ alloy formed by micro-arc oxidation. J Mater Sci. 2015;26:5533.

13. Mishra RS, De PS, Kumar N. Friction Stir Welding and Processing. Berlin, Germany: Springer International Publishing; 2014.

14. Mishra RS, Ma ZY, Charit I. Friction stir processing: a novel technique for fabrication of surface composite. Mater Sci Eng A. 2003;341(1-2): 307-310.

15. Braga DFO, Silva ACFD, Moreira PMGP. 4-Mechanical properties. Advances in Friction-Stir Welding and Processing. Adv Frict-Stir Weld Process. 2014;16:141-197.

16. Wang L, Xie L, Lv Y, et al. Microstructure evolution and superelasticity of layer-like NiTiNb porous metal prepared by eutectic reaction. Acta Mater. 2017;23(1):87.

17. Wang L, Qu J, Chen L, et al. Investigation of deformation mechanisms in? Type Ti-35Nb-2Ta-3Zr alloy via FSP leading to surface strengthening. Metall Mater Trans A. 2015;46(11):4813-4818.

18. Zhu C, Lv Y, Qian C, et al. Proliferation and osteogenic differentiation of rat BMSCs on a novel $\mathrm{Ti} / \mathrm{SiC}$ metal matrix nanocomposite modified by friction stir processing. Sci Rep. 2016;6:38875.

19. Atapour M, Pilchak A, Frankel GS, Williams JC, Fathi MH, Shamanian M. Corrosion behavior of Ti-6Al-4V with different thermomechanical treatments and microstructures. Corros Houston TX. 2010;66(6): 230-240.

20. Atapour M, Pilchak A, Frankel GS, Williams JC. Corrosion behaviour of investment cast and friction stir processed Ti-6Al-4V. Corros Sci. 2010;52(9):3062-3069.

21. Estrin Y, Kasper C, Diederichs S, Lapovok R. Accelerated growth of preosteoblastic cells on ultrafine grained titanium. J Biomed Mater Res A. 2009;90(4):1239-1242.

22. Estrin Y, Ivanova EP, Michalska A, Truong VK, Lapovok R, Boyd R. Accelerated stem cell attachment to ultrafine grained titanium. Acta Biomater. 2011;7(2):900-906.

23. Li G, Cao H, Zhang W, et al. Enhanced osseointegration of hierarchical micro/nano-topographic titanium fabricated by micro-arc oxidation and electrochemical treatment. Acs Appl Mater Interfaces. 2016;8:3840. 
24. Zhang W, Cao H, Zhang X, et al. A strontium-incorporated nanoporous titanium implant surface for rapid osseointegration. Nanoscale. 2016;8(9):5291-5301.

25. Shang Ya-wei ZB-H. Research progress of the biological action of zinc on osteoblasts. Chin J Pract Stomatol. 2013:629-633.

26. Nakashima K, Zhou X, Kunkel G, et al. The novel zinc finger-containing transcription factor osterix is required for osteoblast differentiation and bone formation. Cell. 2002;108(1):17-29.

27. Yu Y, Jin G, Xue Y, Wang D, Liu X, Sun J. Multifunctions of dual Zn/Mg ion co-implanted titanium on osteogenesis, angiogenesis and bacteria inhibition for dental implants. Acta Biomater. 2017;49:590-603.

28. Ding Z, Zhang C, Xie L, et al. Effects of friction stir processing on the phase transformation and microstructure of $\mathrm{TiO} 2$-compounded Ti-6Al-4V alloy[J]. Metallurgical \& Materials Transactions A. 2016;47(12):5675-5679.

29. Wang L, Wang C, Dunand DC. Microstructure and strength of NiTi-Nb eutectic braze joining NiTi wires. Metall Mater Trans A. 2015;46(4): 1433-1436.

30. Lv Y, Wang L, Han Y, Xu X, Lu W. Investigation of microstructure and mechanical properties of hot worked NiAl bronze alloy with different deformation degree. Mater Sci Eng A. 2015;643:17-24.

31. Qian C, Zhu C, Yu W, Jiang X, Zhang F. High-fat diet/low-dose streptozotocin-induced type 2 diabetes in rats impacts osteogenesis and Wnt signaling in bone marrow stromal cells. PLoS One. 2015;10(8): e0136390.

32. Li B, Shen Y, Hu W. Surface nitriding on Ti-6Al-4V alloy via friction stir processing method under nitrogen atmosphere. Appl Surf Sci. 2013;274:356-364.

33. Li B, Shen Y, Luo L, Hu W. Fabrication of TiCp/Ti-6Al-4V surface composite via friction stir processing (FSP): process optimization, particle dispersion-refinement behavior and hardening mechanism. Mater Sci Eng A. 2013;574:75-85.

34. Park CH, Lee CS, Kim YJ, Jang JH, Suh JY, Park JW. Improved preosteoblast response and mechanical compatibility of ultrafine-grained Ti-13Nb-13Zr alloy. Clin Oral Implants Res. 2011;22:735-742.

35. Ahmed T, Rack HJ. Phase transformations during cooling in $\alpha+\beta$ titanium alloys. Mater Sci Eng A. 1998;243(1-2):206-211.

36. Liu YG, Li MQ, Liu HJ. Surface nanocrystallization and gradient structure developed in the bulk TC4 alloy processed by shot peening. J Alloys Compd. 2016;685:186-193.

37. Liu YG, Li MQ, Liu HJ. Nanostructure and surface roughness in the processed surface layer of Ti-6Al-4V via shot peening. Mater Charact. 2017;123:83-90.

38. Zhou X, Chen C. Strengthening and toughening mechanisms of amorphous/ amorphous nanolaminates. Int J Plasticity. 2016;80:75-85.
39. Liang XQ, Zhang JY, Wang YQ, et al. Tuning the size-dependent He-irradiated tolerance and strengthening behavior of crystalline/ amorphous Cu/Ta nanostructured multilayers. Mater Sci Eng A. 2016; 672:153-160.

40. Fan Z, Xue S, Wang J, Yu KY, Wang H, Zhang X. Unusual size dependent strengthening mechanisms of $\mathrm{Cu}$ /amorphous $\mathrm{CuNb}$ multilayers. Acta Mater. 2016;120:327-336.

41. Lin Z, Wang L, Xue X, Lu W, Qin J, Zhang D. Microstructure evolution and mechanical properties of a $\mathrm{Ti}-35 \mathrm{Nb}-3 \mathrm{Zr}-2 \mathrm{Ta}$ biomedical alloy processed by equal channel angular pressing (ECAP). Mater Sci Eng C. 2013;33(8):4551-4561.

42. Ibrahim KM, Hussein AH, Abdelkawy M. Effect of Si-addition as a grain refiner on microstructure and properties of Ti-6Al-4V alloy. Trans Nonferrous Met Soc China. 2013;23(7):1863-1874.

43. Lütjering G, Williams JC. Titanium. Berlin Heidelberg: Springer; 2007.

44. Salasznyk RM, Williams WA, Boskey A, Batorsky A, Plopper GE. Adhesion to vitronectin and collagen I promotes osteogenic differentiation of human mesenchymal stem cells. Biomed Res Int. 2004;2004: 24-34.

45. Zhang W, Li Z, Huang Q, et al. Effects of a hybrid micro/nanorod topography-modified titanium implant on adhesion and osteogenic differentiation in rat bone marrow mesenchymal stem cells. Int $J$ Nanomedicine. 2013;8:257-265.

46. Jin G, Cao H, Qiao Y, Meng F, Zhu H, Liu X. Osteogenic activity and antibacterial effect of zinc ion implanted titanium. Colloids Surf B Biointerfaces. 2014;117:158.

47. Qiao Y, Zhang W, Tian P, et al. Stimulation of bone growth following zinc incorporation into biomaterials. Biomaterials. 2014;35:6882-6897.

48. Huo K, Zhang X, Wang H, Zhao L, Liu X, Chu PK. Osteogenic activity and antibacterial effects on titanium surfaces modified with Zn-incorporated nanotube arrays. Biomaterials. 2013;34:3467-3478.

49. Xu J, Li Z, Hou Y, Fang W. Potential mechanisms underlying the Runx2 induced osteogenesis of bone marrow mesenchymal stem cells. Am J Transl Res. 2014;7:2527.

50. Komori T. Regulation of bone development and maintenance by Runx2 . Front Biosci A J Virtual Libr. 2008;13:898-903.

51. Gang L, Yun-Yu H, Jian-Ning Z, Su-Jia W, Guang-Xin Z. The cellular mechanism of stimulative effects of Collagen I on rabbit bone marrow stromal cells(BMSCs) adhesion. Chin J Orthop Trauma. 2006;8:549-552.

52. Wang G, Li J, Zhang W, et al. Magnesium ion implantation on a micro/ nanostructured titanium surface promotes its bioactivity and osteogenic differentiation function. Int J Nanomed. 2014;9:2387. 


\section{Supplementary material}
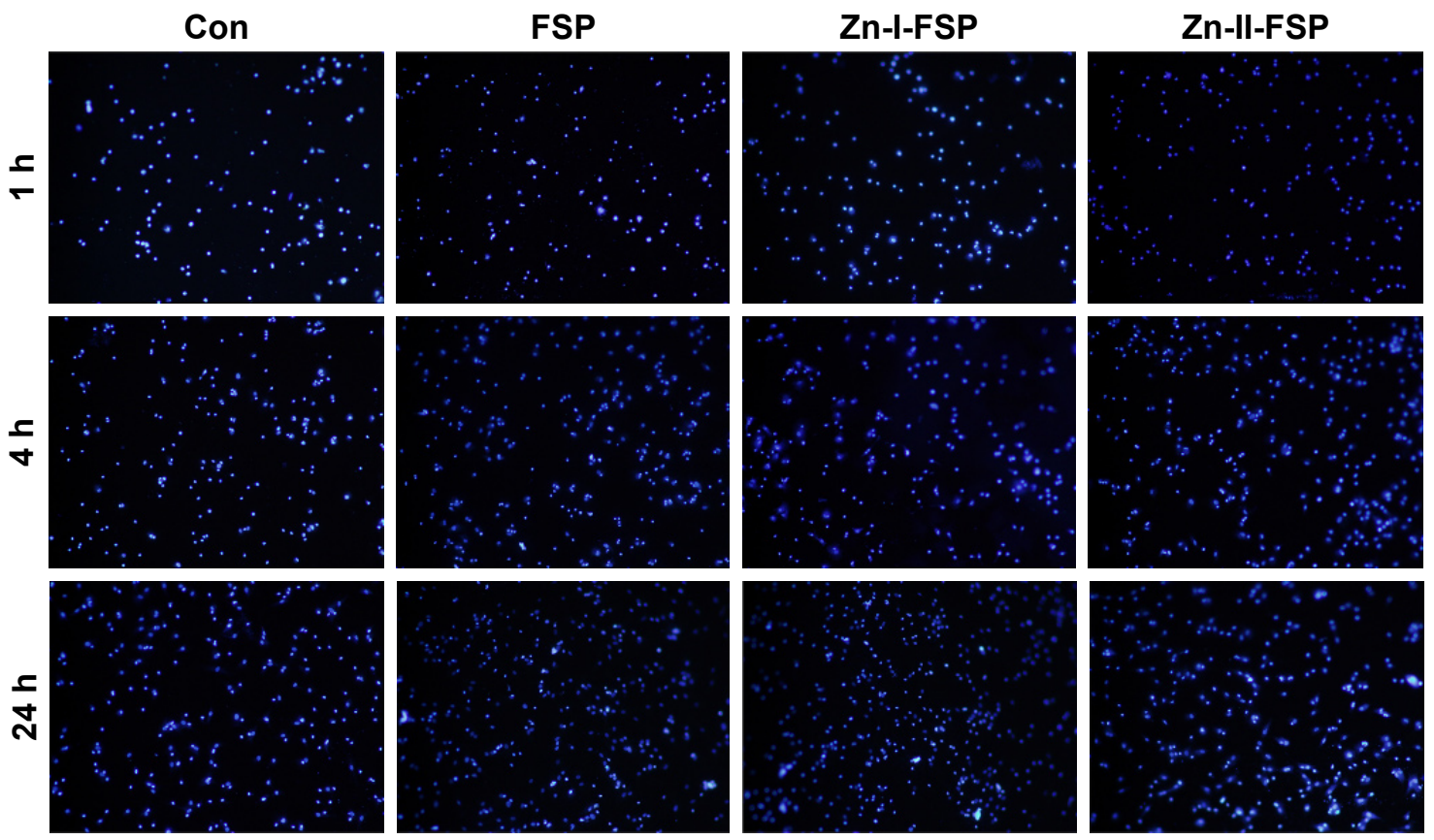

Figure SI Nuclear staining of each group at I, 4, and $24 \mathrm{~h}$.

Abbreviations: Con, control group; FSP, friction stir processing; $\mathrm{Zn}$, zinc.

\section{Publish your work in this journal}

The International Journal of Nanomedicine is an international, peerreviewed journal focusing on the application of nanotechnology in diagnostics, therapeutics, and drug delivery systems throughout the biomedical field. This journal is indexed on PubMed Central, MedLine, CAS, SciSearch ${ }^{\circledR}$, Current Contents ${ }^{\circledR} /$ Clinical Medicine,
Journal Citation Reports/Science Edition, EMBase, Scopus and the Elsevier Bibliographic databases. The manuscript management system is completely online and includes a very quick and fair peer-review system, which is all easy to use. Visit http://www.dovepress.com/ testimonials.php to read real quotes from published authors. 